\title{
An Axiomatization of Cumulative Prospect Theory
}

\author{
PETER WAKKER \\ University of Leiden, Medical Decision Making Unit, Leiden, The Netherlands \\ AMOS TVERSKY* \\ Stanford University, Department of Psychology, Stanford, CA 94305, USA
}

Key words: prospect theory, rank-dependence, sign-dependence, comonotonicity, risk aversion, diminishing marginal utility

\begin{abstract}
This paper presents a method for axiomatizing a variety of models for decision making under uncertainty, including Expected Utility and Cumulative Prospect Theory. This method identifies, for each model, the situations that permit consistent inferences about the ordering of value differences. Examples of rankdependent and sign-dependent preference patterns are used to motivate the models and the "tradeoff consistency" axioms that characterize them. The major properties of the value function in Cumulative Prospect Theory-diminishing sensitivity and loss aversion-are contrasted with the principle of diminishing marginal utility that is commonly assumed in Expected Utility.
\end{abstract}

Attempts to explain decisions under uncertainty that violate Expected Utility have generated several new ideas. (For a survey, see Fishburn 1988.) One notion is that the objects of choice are prospects, defined in terms of gains and losses relative to neutral reference point, rather than acts defined in terms of final asset positions. This notion, first proposed by Markowitz (1952), is the cornerstone of Kahneman and Tversky's (1979) prospect theory. The significance of the reference point stems from the observations that people are generally risk averse for gains, risk seeking for losses, and that losses loom larger than gains. Another empirical generalization that is incorporated into prospect theory is the tendency to overweight small probabilities and to underweight high probabilities. It is natural to model this phenomenon by introducing decision weights obtained from a nonadditive transformation of the probability scale. The problem then arises how to calculate expectation with respect to a nonadditive measure. Several authors (Weymark, 1981; Quiggin, 1982; Schmeidler, 1989; Yaari, 1987) proposed a solution, which reduces to the Choquet functional (Choquet, 1953-4). This model transforms cumulative rather than individual probabilities; we call it the cumulative model. ${ }^{1}$

*The research of the first author has been made possible by a fellowship of the Royal Netherlands Academy of Arts and Sciences. This work was supported by Grant No. 89-0064 from the Air Force Office of Scientific Research to the second author. 
It has been recognized by several authors (Starmer and Sugden, 1989; Luce and Fishburn, 1991; Tversky and Kahneman, 1992) that the basic elements of prospect theory are compatible with the cumulative functional, and that it is possible to construct a model that combines these approaches to produce what we call Cumulative Prospect Theory. This paper presents an axiomatization of Cumulative Prospect Theory, which extends and generalizes the formal development in Tversky and Kahneman (1992). The present treatment differs from Luce and Fishburn (1991) in that it does not involve an operation of joint receipt, does not require higher-order gambles, and does not restrict the shape of the value function.

The axiomatization presented in this paper is based on the tradeoff consistency approach developed by Wakker (1989) and Tversky, Sattath, and Slovic (1988). It provides a unified procedure for axiomatizing Expected Utility and generalized expectation models, and is illustrated in Section 1. The key idea is that different models are characterized by the particular situations in which the ordering of value differences can be inferred from preferences. For example, an inference about the ordering of value differences that is valid in Expected Utility is not valid in the cumulative model unless the prospects in question are comonotonic, i.e., induce the same (desirability) ordering of states of nature. Likewise, an inference about value differences in Cumulative Prospect Theory requires, in addition, that the critical outcomes will have the same sign, i.e., are all gains or all losses. We show that a natural characterization of Cumulative Prospect Theory is obtained by accepting only these inferences. An aim of this paper has been to bring to the fore the tradeoff consistency approach to axiomatic preference theory. The natural way in which characterizations of earlier theories are extended to Cumulative Prospect Theory, as well as the simplicity of additional specifying conditions (for diminishing marginal utility and loss aversion), provide evidence for the fruitfulness of this approach.

The paper is organized as follows. Section 1 gives examples of preferences that violate Expected Utility and exhibit rank-dependence and sign-dependency. Section 2 presents notations and definitions. The next four sections characterize Expected Utility, Cumulative Utility, Sign-Dependent Expected Utility, and Cumulative Prospect Theory. To demonstrate the unity and generality of the tradeoff-consistency approach, we axiomatize all four models. The first two have already been presented in Wakker (1989); they are used in the characterization of the other two.

A schematic summary of the results appears in Table 7.1. The table shows that CPT concerns two independent generalizations of expected utility, one by rank-dependence, the other by sign-dependence. Rank-dependence permits, for instance, pessimism so that events associated with less favorable outcomes are weighted more heavily. Signdependence permits different decision weights for events associated with gains than for events associated with losses. The elicitation of value difference orderings is not disturbed by rank-dependence whenever the prospects used in the elicitation are comonotonic. The elicitation of value difference orderings is not disturbed by sign-dependence whenever the prospects used in the elicitation are "cosigned", i.e. the critical outcomes all have the same sign; this will be further explained below.

Extensions and generalizations of the results are discussed in Section 8. For instance, it is demonstrated that double matching could have been omitted in Theorem 2.c of Tversky and Kahneman (1992), and the extension to infinite state spaces is given. Section 
9 investigates the characteristics of the value function, and provides a method for comparing the value functions of different decision makers in Cumulative Prospect Theory, analogous to the Arrow-Pratt results for Expected Utility. ${ }^{2}$ Loss aversion is characterized ("losses loom larger than gains"). Proofs are presented in the Appendix.

\section{Examples}

We begin with two examples that serve to illustrate the intuitions behind the models under consideration and to suggest the axioms that are used to characterize these models. It seems useful to discuss some ideas and examples already at this stage, prior to giving a formal development. Suppose a biased coin will be tossed, yielding either heads (state 1) or tails (state 2). A decision maker chooses between prospects, i.e., payments contingent on the result of the toss. Throughout the paper outcomes are interpreted as changes of wealth rather than states of wealth. That is, outcomes correspond to gains and losses defined relative to the status quo or an appropriate reference point. Therefore we use the term prospect instead of act, and value function instead of utility function. ${ }^{3}$ Let $\left(x_{1}, x_{2}\right)$ be the prospect yielding $\$ x_{1}$ if state 1 obtains, $\$ x_{2}$ if state 2 obtains.

Example 1.1: Rank-dependence. Consider the following pattern of preferences:

$$
\begin{aligned}
& (11,20) \geqslant(10,21) \\
& (31,20)<(30,21) .
\end{aligned}
$$

A possible interpretation of the shift of preference from (1.1) to (1.2) is as follows. Receiving $\$ 11$ instead of $\$ 10$, denoted by [11; 10], contingent on state 1 , outweighs [21; $20]$, contingent on state 2 . However, $[31 ; 30]$ contingent on state 1 does not outweigh [21; 20], contingent on state 2 . Apparently [11; 10] has more impact than [31;30]. We summarize the inference made from (1.1) and (1.2) by writing [11;10] $>^{*}[31 ; 30]$. Applying Expected Utility (EU) gives

$$
\pi_{1} v(11)+\pi_{2} v(20) \geq \pi_{1} v(10)+\pi_{2} v(21)
$$

and

$$
\pi_{1} v(31)+\pi_{2} v(20)<\pi_{1} v(30)+\pi_{2} v(21),
$$

where $\pi_{1}$ and $\pi_{2}$ are the decision maker's probabilities for states 1 and 2 . It follows readily that $v(11)-v(10)>v(31)-v(30)$. Under EU therefore, $>^{*}$ reveals orderings of value differences. Suppose that we also observe the preferences

$$
\begin{aligned}
& (40,31) \geqslant(41,30) \\
& (40,11) \leqslant(41,10) .
\end{aligned}
$$


These preferences reveal the opposite orderings of value differences, i.e., $v(31)-v(30)$ $\geq v(11)-v(10)$. Thus a contradiction has resulted under EU, and the above four preferences violate $\mathrm{EU}$. We shall see below that there is a sense in which all violations of $\mathrm{EU}$ are reducible to this form. ${ }^{4}$

This paper investigates generalizations of EU in which the inequality $v(11)-v(10)>$ $v(31)-v(30)$ cannot be inferred from the preferences (1.1) and (1.2). All the models that we investigate are special cases of Cumulative Prospect Theory (CPT). The first is Schmeidler (1989) and Gilboa's (1987) Cumulative Utility (CU), which allows a decision maker to pay more attention to a state depending, for example, on whether or not it yields the least desirable outcome in a given prospect. Such behavior might be associated with pessimism. Note that in (1.1) state 2 yields the most desirable outcomes, whereas in (1.2) it yields the least desirable outcomes. A decision maker who pays more attention to the less desirable state, will assign more weight to state 2 in (1.2) than in (1.1). This may explain the shift in preference. In this case it is not possible to infer $v(11)-v(10)>$ $v(31)-v(30)$ because the weights attached to the two states are not the same in (1.1) as in (1.2). Thus the two pairs of preferences no longer yield contradictory inequalities. The inference $v(31)-v(30)>v(11)-v(10)$ remains valid because state 2 yields the least desirable outcome in both (1.3) and (1.4).

Under Cumulative Utility (CU), inferences about orderings of value differences are valid only if the desirability ordering of the states is the same for all prospects under consideration, i.e., if these prospects are comonotonic. In this case it is ensured that for each state the weight assigned by the decision maker is the same for all prospects.

We turn now to another pattern of preference, involving both gains and losses.

Example 1.2: Sign-dependence. Consider the following preferences:

$$
\begin{aligned}
(-2,-9) & \geqslant(-4,-6) \\
(4,-9) & \leqslant(2,-6) \\
(10,4) & \geqslant(12,2) \\
(10,-2) & <(12,-4)
\end{aligned}
$$

The first two preferences yield $[-2 ;-4] \geqslant *[4 ; 2]$; the second pair yields $[4 ; 2]>*[-2$; $-4]$. Under EU, the former implies the inequality $v(-2)-v(-4) \geq v(4)-v(2)$, whereas the latter implies the reversed inequality; consequently, EU is violated. The above preferences, however, can be accommodated by CPT, which suggests risk aversion for gains and risk seeking for losses. Under CPT it will not be possible to infer either inequality from the given preferences because the weights attached to state 1 are not the same in (1.5) where the outcomes are negative as in (1.6) where the outcomes are positive; the same argument applies to state 2 in the preferences (1.7) and (1.8). It is noteworthy that $\mathrm{CU}$ cannot account for these preferences. In this case $\mathrm{CU}$, like EU, yields contradictory orderings of value differences because the outcomes in state 1 are always more desirable than in state 2. Under CPT we permit inferences about orderings of value differences only if all prospects are not only comonotonic but also have the same 
sign profile, i.e., for each state the outcomes are either all nonnegative, or all nonpositive. Only when both conditions are met is it ensured that for each state the weight assigned by the decision maker is the same for all prospects.

The informal discussion of the above examples has suggested the axioms that are needed to characterize the respective models. These axioms will be formulated in the following sections. Because this article brings together four different models, a somewhat heavy notation must be adopted, using indexes for the decision weights and the *-binary relations introduced below to distinguish among the models. In contexts where not all these models are involved, it is possible to simplify the notation by eliminating some of the indices, as in Section 9 below.

\section{Basic concepts}

Let $\mathrm{S}=\{1, \ldots, n\}$ be a finite state space. Subsets of $\mathrm{S}$ are called events. $X$ is a set of outcomes. We assume that $X$ is a connected topological space (e.g., $X=I R$; for topological definitions, see for instance Kelley, 1955). $\mathscr{F}$ denotes the set of prospects, i.e., functions from $S$ to $X$. We often identify $\mathscr{F}$ with $X^{n}$, so that $\left(x_{1}, \ldots, x_{n}\right)$ denotes the prospect assigning outcome $x_{j}$ to state $j$ for each $j$. Given a prospect $f$, an outcome $x$, and a state $j$, we use $x_{j} f$ to denote the prospect $f$ with $f(j)$ replaced by $x$. $\mathscr{F}$ is endowed with the product topology. In this paper outcomes describe changes with respect to a status quo or an appropriate reference level. To underscore this interpretation we use the term prospect instead of Savage's term act. For an outcome $x \in X, \bar{x}$ denotes the constant prospext ( $x$, $\ldots, x)$. The status quo is an element of $X$, denoted by 0 . We assume in this paper that the status quo is fixed, i.e., we restrict attention to preferences in relation to one status quo. The question concerning variations in the status quo lies beyond the scope of the present paper.

Let $\geqslant$ denote a preference relation on $\mathscr{F}$. As usual $>$ denotes the asymmetric part, $\sim$ the symmetric part, and $\leqslant, \prec$ denote reversed preferences. We shall use the same notations for the binary relations on $X$ derived through constant prospects. That is, $x \geqslant y$ if and only if $\bar{x} \geqslant \bar{y}$. An outcome $x>0$ is positive, an outcome $x<0$ is negative. Let $X^{+}=$ $\{x \in X: x \geqslant 0\}$ and $X^{-}=\{x \in X: x \leqslant 0\}$.

Below we provide standard definitions of additive conjoint measurement. In the generalizations of EU studied in this article, additivity is typically satisfied only on specific subsets of $\mathscr{F}$. The shapes of these subsets are characteristic of particular generalizations of EU. Standard conditions, such as independence, will be satisfied only on these subsets; also the definition of essential states should be restricted to those subsets. Hence we shall generalize the standard definitions of additivity to subsets $F$ of $\mathscr{F}$. A function $V$ represents $\geqslant$ on $F$ if, for all $f, g \in F, f \geqslant g$ if and only if $V(f) \geq V(g)$. If $\geqslant$ is represented by a function on $F$, then $\geqslant$ is a weak order on $F$, i.e., it is complete on $F(f \geqslant \mathrm{~g}$ or $g \geqslant f$ for all $f, g \in F)$ and transitive on $F(f \geqslant g$ and $g \geqslant h$ then $f \geqslant h$ for all $f, g, h \in F)$. If $V$ is continuous on $\mathrm{F}$ then $\geqslant$ is also continuous, i.e., $\{f \in F: f \geqslant g\}$ and $\{f \in F: f \leqslant g\}$ are closed subsets of $F$ for each $g \in F$.V is (continuously) ordinal if it is unique up to a continuous 
strictly increasing transformation. It is an interval scale if it is unique up to a positive affine transformation, i.e., up to multiplication by a positive constant, and addition of a real number. It is a ratio-scale if it is unique up to multiplication by a positive constant.

$V$ is an additive representation on $F$ if there exist real-valued functions $V_{1}, \ldots, V_{n}$ such that $V(f)=\sum_{j=1}^{n} V_{j}(f(j))$ for all $f \in F$; obviously the domains of the $V_{j}$ 's must contain all values $f(j)$ for $f \in F$. If an additive $V$ represents $\geqslant$ on $F$, then the $V_{j}$ 's are called additive value functions on $F$.

An event $A$ is inessential on $F$, or $F$ inessential for short, if $f \sim g$ for all $f, g$ in $F$ that agree outside $A$; an event is essential on $F$ if it is not inessential on $F$. We call $\geqslant$ weakly monotonic on $F$ if, for all $f, g \in F, f \geqslant g$ whenever $f(j) \geqslant g(j)$ for all $j$; $\geqslant$ is strongly monotonic on $F$ if, for all $f, g \in F, f>g$ whenever $f(j) \geqslant g(j)$ for all $j$ and $f(i)>g(i)$ for some $F$ essential $i$. In this article, the expression "on $\mathscr{F}$ ' is usually omitted.

A capacity $W$ on $S$ is a function on $2^{S}$ such that $W(\emptyset)=0, W(S)=1$, and $A \supset B \Rightarrow$ $W(A) \geq W(B)$. Obviously, if a capacity $W$ is additive, i.e., $W(A \cup B)=W(A)+W(B)$ for all disjoint events $A, B$, then $W$ is a probability measure. For a capacity $W$ on $S$, and a function $h: S \rightarrow I R$, the Choquet integral, denoted by $\int_{S} h d W$, or $\int_{S} h(i) d W$, is

$$
\int_{I R^{+}} W(\{i: h(i) \geq t\}) d t+\int_{I R^{-}}[W(\{i: h(i) \geq t\})-1] d t .
$$

We next describe the subsets of $f$ on which the various models are additive. For EU this is all of $\mathscr{F}$. Next we turn to CU. Let o be a permutation on $\{1, \ldots, \mathrm{n}\}$, and let $\mathscr{F} \mathrm{O}:=\{f \in$ $\mathscr{F}: f(\mathrm{o}(1)) \leqslant \ldots \leqslant f(\mathrm{o}(n))\}$; these are the additive subsets in CU. For Sign-Dependent Expected Utility, introduced below, let $\mathscr{F}^{+}$be the set of nonnegative prospects, and let $f^{-}$be the set of nonpositive prospects. For a prospect $f$ define $f^{+}$and $f^{-}$in the usual way, i.e., $f^{\vdash}(s)=f(s)$ if $f(s) \geqslant 0, f^{+}(s)=0$ if $f(s)<0, f^{-}(s)=f(s)$ if $f(s) \leqslant 0, f^{-}(s)=0$ if $f(s)$ $>0$. We say that a prospect $f$ lives on event $A$ if $f(j) \sim 0$ for all $j \in A^{c .5}$ its positive (negative) part lives on $A$ if $f^{+}\left(f^{-}\right)$lives on $A$, i.e., if $f(j) \leqslant 0(f(j) \geqslant 0)$ for all $j \in A^{c}$. Let $\mathscr{F}^{A}$ be the set of prospects for which the positive part lives on $A$, the negative part on $A^{c}$; these are the additive subsets in the Sign-Dependent Expected Utility model. We combine superscripts to denote intersections. For example $\mathscr{F}^{A o+}=\mathscr{F} \mathscr{A} \cap \mathscr{P}^{\circ} \cap \mathscr{F}^{+}=$ $\left\{f \in \mathscr{F}: f(\mathrm{o}(1)) \leqslant \ldots \leqslant f(\mathrm{o}(n)), f=f^{+}, f^{+}\right.$lives on $\left.A\right\}$. The sets $\mathscr{F}^{A o}$ are the additive subsets for CPT.

Each of the representations developed in this paper can be expressed as a weighted sum of the form $\sum \pi_{j} v(f(j))$, where the $\pi_{j}$ 's are called decision weights. The $\pi_{j}$ 's are often endowed with superscripts.

The following structural conditions are assumed throughout the paper.

Structural Assumption 2.1: $\mathrm{S}=\{1, \ldots, n\}$ is a finite state space, $\mathscr{F}=X^{n}$ is the set of prospects endowed with a connected product topology, and $\geqslant$ is a binary relation on $\mathscr{F}$.

\section{Expected utility}

Under Expected Utility (EU) there exists a probability measure $P$ on $S$, and a value function $v: X \rightarrow I R$, such that 


$$
V: f \mapsto \int_{S} v(f(s)) d P
$$

represents preferences. This can be rewritten as the weighted sum

$$
\sum \pi_{j} v(f(j))
$$

with

$$
\pi_{\mathrm{j}}=P(j)
$$

for each $j$. The model is additive; hence the usual definitions of additive conjoint measurement can be adopted. Note that $\pi_{j}>0$ if and only if $x_{j} f>y_{j} f$ for some outcomes $x, y$ and a prospect $f$, i.e., if and only if $j$ is essential. Here essentiality is taken on all of $F$, as in the standard treatment of additive conjoint measurement.

The following * relations are useful for both elicitation and characterization. Define $[x ; y]>^{*}\left[x^{\prime} ; y^{\prime}\right]$, or $x y>^{*} x^{\prime} y^{\prime}$ for short, if there exist prospects $f, g$, and a state $j$, such that

$$
\begin{aligned}
& x_{j} f \geqslant y_{j} g \text { and } \\
& x_{j}^{\prime} f<y_{j}^{\prime} g
\end{aligned}
$$

We write $\geqslant *$ instead of $>^{*}$ if in the lower preference we have $\leqslant$ instread of $<$ and $j$ is essential. The * relations in general do not have to satisfy natural properties, e.g. $>*$ does not have to be asymmetric, and $>*$ does not have to be the asymmetric part of $\geqslant *$. As usual, $\leqslant^{*}, \prec^{*}$ denote the reversed relations. The following lemma shows that under EU these relations are indeed suited to elicit the function $v$. While elementary, the result is central for this paper; hence we display it as a lemma and give its proof.

Lemma 3.1. If EU holds then:

$$
\begin{aligned}
& x y>^{*} x^{\prime} y^{\prime} \Rightarrow v(x)-v(y)>v\left(x^{\prime}\right)-v\left(y^{\prime}\right), \\
& x y \geqslant * x^{\prime} y^{\prime} \Rightarrow v(x)-v(y) \geq v\left(x^{\prime}\right)-v\left(y^{\prime}\right) .
\end{aligned}
$$

Proof. Consider (3.3). We substitute EU in (3.1) and (3.2), to get

$$
\begin{aligned}
& \sum_{i \neq j} \pi_{i} v(f(i))+\pi_{j} v(x) \geq \sum_{i \neq j} \pi_{i} v(g(i))+\pi_{j} v(y) \\
& \sum_{i \neq j} \pi_{i} v(f(i))+\pi_{j} v\left(x^{\prime}\right)<\sum_{i \neq j} \pi_{i} v(g(i))+\pi_{j} v\left(y^{\prime}\right) .
\end{aligned}
$$

This implies $\pi_{j} v(x)-\pi_{j} v(y)>\pi_{j} v\left(x^{\prime}\right)-\pi_{j} v\left(y^{\prime}\right)$. Thus, by cancelling the evidently positive $\pi_{j}$, we get $v(x)-v(y)>v\left(x^{\prime}\right)-v\left(y^{\prime}\right)$. The same reasoning applies to (3.4), except that positivity of $\pi_{j}$ (i.e., essentiality of state $j$ ) does not follow from the preferences, so it has to be assumed. 
The following condition is necessary for EU:

Definition 3.2. Tradeoff consistency is satisfied if there are no outcomes $x, y, x^{\prime}, y^{\prime}$ such that both $x y>^{*} x^{\prime} y^{\prime}$ and $x y \leqslant{ }^{*} x^{\prime} y^{\prime}$ hold.

Wakker (1989, Theorem IV.2.7 and Remark A3.1) showed that this condition is also sufficient for EU in the presence of the usual assumptions.

Theorem 3.3. Suppose that the Structural Assumption 2.1 holds, and that at least two states are essential. Then EU with continuous value function holds if and only if $\geqslant$ satisfies the following conditions:

(a) weak ordering;

(b) continuity; and

(c) tradeoff consistency.

Further, the probability measure is uniquely determined, and the value function is an interval scale.

\section{Cumulative utility (CU)}

Cumulative utility was initiated by Schmeidler (1989); see also Gilboa (1987). The term "cumulative utility" was introduced in Tversky and Kahneman (1992). Another common term is "Choquet expected utility." Wakker (1990a) showed that cumulative utility can be applied to decision making under risk (where choice alternatives are probability distributions over outcomes, instead of acts or prospects), and then, under the natural assumption of stochastic dominance, is identical to rank-dependent utility as introduced by Quiggin (1982).

A central concept in cumulative utility is comonotonicity. A set of prospects $F$ is comonotonic if for no $f, g \in F, i, j \in S, f(i)>f(j)$ and $g(i)<g(j)$. It follows readily (see, e.g., Wakker, 1989, Lemma VI.3.3) that $F$ is comonotonic if and only if $F \subset$ go for some permutation o on $\{1, \ldots, n\}$. Hence the sets $\mathscr{F 0}$ are maximal comonotonic sets. As we shall see, $\mathrm{CU}$ gives additive representations within maximal comonotonic sets; hence some of the conditions will be restricted to these sets. We say that $\mathrm{CU}$ holds if there exists a capacity $W$ and a value function $v: X \rightarrow I R$, such that

$$
V: f \mapsto \int_{S} v(f(i)) d W
$$

represents preferences. This can be rewritten as the weighted sum

$$
\sum_{j=1}^{n} \pi_{j}^{c} v(f(j))
$$

as follows. For prospect $f$ we take a permutation o such that $f(\mathrm{o}(1)) \leqslant \ldots \leqslant f(\mathrm{o}(n))$ and define

$$
\pi_{o(j)}^{c}=W(\{\mathrm{o}(j), \ldots, \mathrm{o}(n)\})-W(\{\mathrm{o}(j+1), \ldots, \mathrm{o}(n)\})
$$


for each $j$. Dependency on o is expressed by the superscript $c$ (for comonotonicity). If $f(j)$ $\sim f(i)$ for some $i \neq j$, then the ordering of states $i$ and $j$ by o can be chosen arbitrarily, and the decision weights $\pi_{j}^{c}$ depend on this arbitrary choice. In that case it should be specified for $\pi_{j}^{c}$ which o is chosen. Usually no misunderstandings will arise, and, as is easily seen, the particular choice of o is immaterial for the end result in (4.1). Hence we usually do not indicate the dependence of the decision weights $\pi_{j}^{c}$ on the permutation o induced by $f$.

Note that for comonotonic prospects the same o can be chosen. Furthermore, $\pi_{\mathrm{o}(j)}^{c}>$ 0 if and only if $o(j)$ is essential on the maximal comonotonic set with respect to 0 . A state can be essential on one maximal comonotonic set, but inessential on another.

CU generalizes EU by allowing the decision weights of states to depend on the desirability ordering of the states induced by a given prospect. Indeed, (4.2) expresses the dependence on the set of states that yield an equivalent or better outcome. Such events are called cumulative. CU allows for some forms of optimism or pessimism (see Example 1.1). The pattern of preferences in this example is obtained by setting $W(\{1\})=W(\{2\})$ $=1 / 3, v(x)=x$ for $x \leq 20, v(x)=2 x-20$ for $20 \leq x \leq 30, v(x)=3 x-50$ for $30 \leq x \leq$ $40, v(x)=4 x-90$ for $40 \leq x$.

To characterize the model, the * relations are modified as follows. We write $x y>{ }_{c}^{*} x^{\prime} y^{\prime}$ if (3.1) and (3.2) hold, provided the four prospects in question $\left\{x_{j} f, y_{j} g, x_{j}^{\prime} f, y_{j}^{\prime} g\right\}$ are comonotonic. Analogously we write $x y \geqslant{ }_{c}^{*} x^{\prime} y^{\prime}$ if (3.1) and (3.2) hold with weak instead of strict preference in (3.2), provided the four prospects in question are comonotonic and $j$ is essential on a maximal comonotonic set containing these prospects. The following lemma shows that under $\mathrm{CU}$ these relations reveal the ordering of value differences:

Lemma 4.1. If CU holds then:

$$
\begin{aligned}
& x y>_{c}^{*} x^{\prime} y^{\prime} \Rightarrow v(x)-v(y)>v\left(x^{\prime}\right)-v\left(y^{\prime}\right), \\
& x y \geqslant_{c}^{*} x^{\prime} y^{\prime} \Rightarrow v(x)-v(y) \geq v\left(x^{\prime}\right)-v\left(y^{\prime}\right) .
\end{aligned}
$$

Proof. The proof is identical to the proof of Lemma 3.1, only now the decision weights $\pi_{i}$ and $\pi_{j}$ are replaced by $\pi_{i}^{c}$ and $\pi_{j}^{c}$, respectively. Comonotonicity guarantees that the same set of decision weights $\pi_{1}^{c} \ldots \pi_{n}^{c}$ can be used for all prospects in question.

The following condition is necessary for CU:

Definition 4.2. Comonotonic tradeoff consistency is satisfied if there are no outcomes $x$, $y, x^{\prime}, y^{\prime}$ such that both $x y \geqslant_{c}^{*} x^{\prime} y^{\prime}$ and $x y<_{c}^{*} x^{\prime} y^{\prime}$ hold.

Wakker (1989, Theorem VI.5.1 and Remark A3.1) showed that this condition is also sufficient for $\mathrm{CU}$ in the presence of the usual assumptions.

Theorem 4.3. Suppose that the Structural Assumption 2.1 holds, and that there exists an $F^{\circ}$ with at least two $F^{\circ}$ essential states. CU with a continuous value function holds if and only if $\geqslant$ satisfies the following conditions:

(a) weak ordering; 
(b) continuity;

(c) comonotonic tradeoff consistency.

Further, the capacity is uniquely determined, and the value function is an interval scale.

\section{Sign-dependent expected utility (SDEU)}

In this section we present a generalization of EU that allows for sign-dependence, but coincides with EU on $\mathscr{F}^{+}$and on $\mathscr{F}^{-}$. This generalization, called Sign-Dependent Expected Utility ( $S D E U$ ), is of limited interest on its own; it is presented as a preparation for Cumulative Prospect Theory. A set $F$ of prospects is cosigned if there do not exist $f, g \in F$, and a state $j$, such that $f(j)>0,0>g(j) \cdot{ }^{6}$ It is easily seen that $F$ is cosigned if and only if $F \subset \mathscr{F}^{A}$ for some event $A$. Thus sets $\mathscr{F} A$ are maximal cosigned sets. SDEU holds if there exist two probability measures $P^{+}$(associated with gains) and $P^{-}$(associated with losses), and a value function $\mathrm{v}$, such that

$$
V: f \mapsto \int_{S} v\left(f^{+}(i)\right) d P^{+}+\int_{S} v\left(f^{-}(i)\right) d P^{-}
$$

represents preferences. This model satisfies weak monotonicity since an increase in payment of $f$ will increase either $f^{+}$or $f^{-}$or (if the increase is from negative to positive) both, and will decrease neither. For the following expression it is necessary to set $v(0)=$ 0 , as is commonly done in sign-dependent models. We shall assume this throughout the paper for the sign-dependent models. Then (5.1) can be rewritten as

$$
\sum_{j=1}^{n} \pi_{j}^{s} v(f(j))
$$

where $\pi_{j}^{s}$ is $\mathrm{P}^{+}(j)$, also denoted by $\pi_{j}^{+}$, if $f(j)$ is positive, $\pi_{j}^{s}$ is $\mathrm{P}^{-}(j)$, also denoted by $\pi_{j}^{-}$, if $f(j)$ is negative. The choice of $\pi_{j}^{s}$ is immaterial if $f(j)$ is 0 ; it can be either $\pi_{j}^{+}$or $\pi_{j}^{-}$. Note that $\pi_{j}^{+}>0$ if and only if $j$ is essential on a maximal cosigned set where positive outcomes are assigned to state $j$; the same applies to $\pi_{j}^{-}>0$ (with negative outcomes assigned to state $j$ ). Note that a state can be essential for positive outcomes, and inessential for negative outcomes.

To characterize the model, the * relations are modified as follows. We write $x y>_{s}^{*} x^{\prime} y^{\prime}$ if (3.1) and (3.2) hold, provided the four prospects in question $\left\{x_{j} f, y_{j} \mathrm{~g}, x_{j}^{\prime} f, y_{j}^{\prime} g\right\}$ are cosigned. Analogously we write $x y{ }_{s}^{*} x^{\prime} y^{\prime}$ if (3.1) and (3.2) hold with weak instead of strict preference in (3.2), the four prospects in question are cosigned, and $j$ is essential on a maximal cosigned set containing the four prospects. Note that either $x, y, x^{\prime}, y^{\prime}$ are all nonnegative, or nonpositive. The following lemma shows that under SDEU these * relations reveal the ordering of value differences:

Lemma 5.1. If SDEU holds, then:

$$
\begin{aligned}
& x y>{ }_{s}^{*} x^{\prime} y^{\prime} \Rightarrow v(x)-v(y)>v\left(x^{\prime}\right)-v\left(y^{\prime}\right), \\
& x y \geqslant{ }_{s}^{*} x^{\prime} y^{\prime} \Rightarrow v(x)-v(y) \geq v\left(x^{\prime}\right)-v\left(y^{\prime}\right) .
\end{aligned}
$$


Proof. The proof is identical to the proof of Lemma 3.1, only now the decision weights $\pi_{i}$ and $\pi_{j}$ are replaced by $\pi_{i}^{s}$ and $\pi_{j}^{s}$, respectively. Because the prospects in question are cosigned the same decision weights $\pi_{1}^{s} \ldots \pi_{n}^{s}$ can be used for all prospects in question. $\square$

Thus the following condition is necessary for SDEU:

Definition 5.2. Cosigned tradeoff consistency is satisfied if there are no outcomes $x, y, x^{\prime}$, $y^{\prime}$ such that both $x y \geqslant_{s}^{*} x^{\prime} y^{\prime}$ and $x y<{ }_{s}^{*} x^{\prime} y^{\prime}$ hold.

Again, this condition turns out to be sufficient in the presence of the usual conditions. We introduce two additional conditions, which will be further discussed in Section 6. Gain-loss consistency is satisfied if for all $f, g \in \mathscr{F}, f \sim \overline{0}, f^{+} \sim g^{+}$, and $f^{-} \sim g^{-}$implies $g \sim \overline{0}$. We call $\geqslant$ truly mixed if there exists a prospect $f$ with $f^{+}>\overline{0}$ and $\overline{0}>f^{-}$. The following proposition is a straightforward corollary of Theorem 6.3. (Cosigned tradeoff consistency will, by Theorem 3.3, imply additivity of the representation both on $\mathscr{F}^{+}$and on $\mathscr{F}^{-}$, provided these sets have two or more essential states.)

Proposition 5.3. Suppose that the Structural Assumption 2.1 holds and assume the truly mixed case. Then SDEU with continuous value function holds if and only if $\geqslant$ satisfies the following conditions:

(a) weak ordering;

(b) continuity;

(c) gain-loss consistency and cosigned tradeoff consistency.

Further, both probability measures are uniquely determined, and the value function is a ratio scale.

\section{Cumulative prospect theory (CPT)}

This model combines the two preceding generalizations of EU. A set of prospects is sign-comonotonic if it is both cosigned and comonotonic. It is easily seen that a set is sign-comonotonic if and only if it is a subset of a set $\mathscr{F}^{A} \mathrm{o}$. Hence the sets $\mathscr{F} A 0$ are the maximal sign-comonotonic sets. They are of the form $\{f \in \mathscr{F}(\mathrm{o}(1)) \leqslant \ldots \leqslant f(\mathrm{o}(k)) \leqslant 0$ $\leqslant f(\mathrm{o}(k+1)) \leqslant \ldots \leqslant f(\mathrm{o}(n))\}$, for some permutation o, integer $k$, and event $A=$ $\{\mathrm{o}(k+1), \ldots, \mathrm{o}(n)\}$. In CPT we have an additive representation within each set $\mathscr{F} A \mathrm{~A}$; hence such sets $\mathscr{F} 4 \mathrm{o}$ will play a central part in the proofs.

The notation was chosen to simplify the formulation of the model presented and axiomatized in Theorem 6.3. Consequently we integrate for negative outcomes in a way dual to that for positive outcomes, and the formulas will be different than those for $\mathrm{CU}$.

Two capacities $W^{+}$and $W^{-}$are associated with gains and losses respectively. We also use the dual of $W^{-}$, denoted by $\hat{W}^{-}$, and defined by $\hat{W}^{-}(A)=1-W^{-}\left(A^{c}\right), A \subset S$. As usual $v$ denotes a value function. Now preferences are represented by

$$
V: f \mapsto \int_{S} v\left(f^{+}(i)\right) d W^{+}+\int_{S} v\left(f^{-}(i)\right) d \hat{W}^{-} .
$$


Note that for negative outcomes we use the dual of $W^{-}$. Thus $C U$ results if $W^{+}$is the dual of $W^{-}$. The following rewriting of (6.1) shows an appealing feature of the use of the dual capacity for the negative outcomes:

$$
\int_{I R^{+}} W^{+}(\{i: v(f(i)) \geq t\}) d t-\int_{I R^{-}}\left[W^{-}(\{i: v(f(i)) \leq t\})\right] d t .
$$

Without loss of generality we assume $v(0)=0$. Then we can rewrite (6.1) as

$$
\sum_{j=1}^{n} \pi_{j}^{c s} v(f(j))
$$

where the decision weights $\pi_{j}^{c s}$ are defined as follows. For prospect $f$ we take a permutation $o$ on $\{1, \ldots, n\}$ and a $k$ such that $f(o(1)) \leqslant \ldots \leqslant f(o(k)) \leqslant 0 \leqslant f(o(k+1)) \leqslant \ldots \leqslant$ $f(o(n))$. Next we define

$$
\begin{aligned}
& \text { for } \left.j<k: \pi_{\mathrm{o}(j)}^{c s}=\pi_{o(j)}^{c^{-}}\right)=W^{-}(\{\mathrm{o}(1), \ldots, \mathrm{o}(j)\})-W^{-}(\{\mathrm{o}(1), \ldots, \mathrm{o}(j-1)\}), \\
& \text { for } \left.j \geq k: \pi_{o(j)}^{c s}=\pi_{o(j)}^{c^{+}}\right)=W^{+}(\{\mathrm{o}(j), \ldots, \mathrm{o}(n)\})-W^{+}(\{\mathrm{o}(j+1), \ldots, \mathrm{o}(n)\}) .
\end{aligned}
$$

So the decision weights are as in CU, except that for positive $f(j)$ we use $W^{+}$, the gain-capacity, and for negative $f(j)$ we use $\hat{\mathrm{W}}^{-}$, the dual of the loss-capacity. Note that $\left.\pi_{o(j)}^{c s}\right)>0$ if and only if $o(j)$ is essential on the related maximal sign-comonotonic set. Recall that a state can be essential on one maximal sign-comonotonic set, but inessential on another. The comments following (4.2) about potential ambiguity in the notation of the decision weights apply here as well. As noted earlier, we use the double index to distinguish CPT from the other models discussed in the previous section. The double index can be dropped when this model alone is considered, as in Section 9.

To characterize the model, the * relations are modified as follows. We write $x y>_{c s}^{*} x^{\prime} y^{\prime}$ if (3.1) and (3.2) hold, provided the four prospects in question $\left\{x_{j} f, y_{j} g, x_{j}^{\prime} f y_{j}^{\prime}{ }_{j} g\right\}$ are sign-comonotonic. Analogously we write $x y \geqslant_{c s}^{*} x^{\prime} y^{\prime}$ if (3.1) and (3.2) hold with weak instead of strict preference in (3.2), the four prospects in question are sign-comonotonic, and $j$ is essential on a maximal sign-comonotonic set containing these prospects. The following lemma shows that under CPT these relations reveal the ordering of value differences:

Lemma 6.1. If CPT holds, then:

$$
\begin{aligned}
& x y>{ }_{c s}^{*} x^{\prime} y^{\prime} \Rightarrow v(x)-v(y)>v\left(x^{\prime}\right)-v\left(y^{\prime}\right), \\
& x y \geqslant{ }_{c s}^{*} x^{\prime} y^{\prime} \Rightarrow v(x)-v(y) \geq v\left(x^{\prime}\right)-v\left(y^{\prime}\right) .
\end{aligned}
$$

Proof. The proof is identical to the proof of Lemma 3.1, only now the decision weights $\pi_{i}$ and $\pi_{j}$ are replaced by $\pi_{i}^{c s}$ and $\pi_{j}^{c s}$, respectively. Sign-comonotonicity ensures that the same set of decision weights $\pi_{1}^{c s} \ldots \pi_{n}^{c s}$ can be used for all prospects in question.

The following condition is necessary for CPT: 
Definition 6.2. Sign-comonotonic tradeoff consistency is satisfied if there are no outcomes $x, y, x^{\prime}, y^{\prime}$ such that both $x y \geqslant{ }_{c s}^{*} x^{\prime} y^{\prime}$ and $x y<_{c s}^{*} x^{\prime} y^{\prime}$ hold.

We shall only consider the case where tradeoffs between positive and negative outcomes can occur. That is, we assume that $\geqslant$ is truly mixed: there exists a prospect $f$ with both $f^{+}>\overline{0}$ and $\overline{0}>f^{-}$. In the central theorem of this paper we shall also use gain-loss consistency (defined in Section 5). In Section 8 we show that this condition can often be omitted. Gain-loss consistency is somewhat weaker than the double-matching condition used by Tversky and Kahneman (1992), but it is the only implication used in their proof.

The proof of the following result is given in the Appendix:

Theorem 6.3. Suppose the Structural Assumption 2.1 holds, and assume the trulymixed case. Then the following two statements are equivalent:

(i) Cumulative Prospect Theory holds with a continuous value function;

(ii) The preference relation $\geqslant$ satisfies the following conditions:

(a) weak ordering;

(b) continuity;

(c) gain-loss consistency and sign-comonotonic tradeoff consistency.

Further, both capacities are uniquely determined, and the value function is a ratio scale.

\section{Summary of results}

Table 7.1. Orderings of tradeoffs are revealed through preferences $x_{j} f \geqslant y_{j} g$ and either $x^{\prime} j f<y_{j}^{\prime} g$ or $x^{\prime} j \leqslant \leqslant y^{\prime} g^{\prime}$. Columns and rows in the table indicate the restrictions imposed upon the four prospects in question. The theory characterized by tradeoff consistency with respect to the revealed orderings of tradeoffs is given in the appropriate cells. The relevant phenomena that deviate from EU are given in parentheses.

\begin{tabular}{|c|c|c|}
\cline { 2 - 3 } \multicolumn{1}{c|}{} & $\begin{array}{c}\text { cosigned } \\
\text { (sign-dependence) }\end{array}$ & - \\
\hline $\begin{array}{c}\text { comonotonic } \\
\text { (rank-dependence) }\end{array}$ & $\mathrm{CPT}$ & $\mathrm{CU}$ \\
\hline- & $\mathrm{SDEU}$ & $\mathrm{EU}$ \\
\hline
\end{tabular}

\section{Extensions and generalizations}

Theorem 6.3 is the central representation theorem of this paper. Every preference relation satisfying the conditions of further theorems below also satisfies the conditions of Theorem 6.3. However, as demonstrated below, some of the assumptions of Theorem 6.3 can be weakened in special cases because they are implied by the other assumptions. Let us also mention that the outcome-dependent capacity form, characterized in Chew 
and Wakker (1991), is more general than CPT. For decision making under risk, this model was presented in Green and Jullien (1988), Chew and Epstein (1989), Chateauneuf (1990), Segal (1993), and was also discussed in Wakker (1993c).

\subsection{Omitting gain-loss consistency in theorem 6.3}

Gain-loss consistency is often implied by the other conditions, it can sometimes be omitted. In the proof of the following observation, given in Appendix A3, somewhat more general results are given. Here we only give the most accessible result.

Observation 8.1. Let $n \geq 3$. Suppose the following monotonicity condition is satisfied: $[\forall i: f(i) \geqslant g(i), \exists i: f(i)>g(i)] \Rightarrow f>g$. Then in Theorem 6.3 the condition of gain-loss consistency can be omitted.

Thus in Theorem 2.c of Tversky and Kahneman (1992), the condition of double matching can be omitted. For $n=2$ the result of the above observation does not hold, as Figure 1 shows. It also illustrates that gain-loss consistency cannot be omitted in Theorem 6.3.

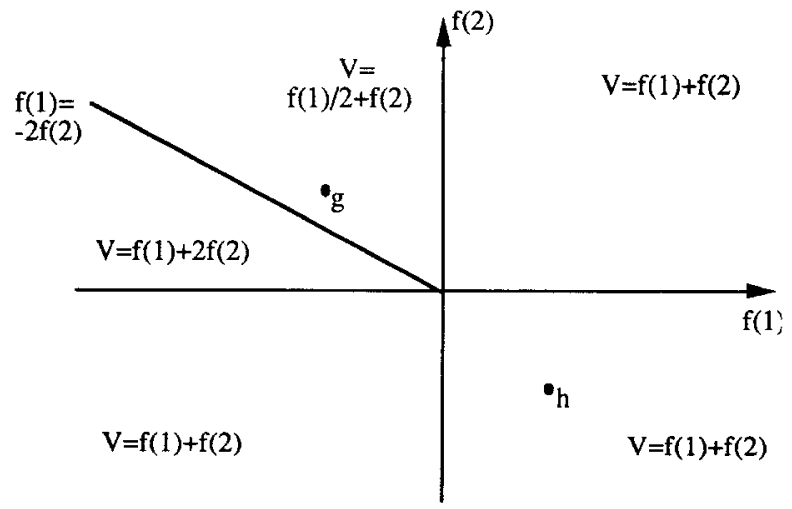

Figure 1. (Necessity of gain-loss consistency). Preferences are represented by the function $V$. Gain-loss consistency is violated: with $-g(1)=g(2)=h(1)=-h(2)=2$, we have $g^{+} \sim h^{+}, g^{-} \sim h^{-}$, but $g>h$. So CPT is violated. A CPT - representation can be established on the first three quadrants, by setting $W(\{1\})=W(\{2\})=$ $1 / 2$, and $v=$ identity. A CPT-representation can also be established on the first, third, and fourth quadrant, by setting $W(\{1\})=W(\{2\})=1 / 2, v$ is identity on $I R^{-}$, and $v$ is twice identity on $I R^{+}$. In particular on all sign-comonotonic sets $F^{A 0}$ we have a CPT representation. Also, if $x, y, x^{\prime}, y^{\prime}$ are of a same sign, then $[x ; y] \geqslant_{s}^{*}$ $\left[x^{\prime} ; y^{\prime}\right]$ if and only if $x-y \geq x^{\prime}-y^{\prime}$, so that sign-comonotonic (even cosigned) tradeoff consistency holds. All conditions of Statement (ii) in Theorem 6.3, execpt gain-loss consistency, are satisfied. 


\subsection{A weakening of sign-comonotonic tradeoff consistency: Characterizing CPT as EU} within each sign-comonotonic subset

Under monotonicity conditions, slightly stronger than those in Observation 8.1, another generalization is possible. It then suffices to obtain EU within each sign-comonotonic subset. To formulate the result, we define $x y>{ }_{A 0}{ }^{\prime} x^{\prime} y^{\prime}$ if there exist prospects $f, g$, and a state $j$, such that $x_{j} f \geqslant y_{j} g$ and $x_{j}^{\prime} f\left\langle y_{j}^{\prime} g\right.$, and $x_{j} f, y_{j} g, x_{j}^{\prime} f, y_{j}^{\prime} g \in \mathscr{F}^{\prime} A 0$. So this is like $x y>$ ${ }_{c s}^{*} x^{\prime} y^{\prime}$, only now the sign-comonotonic set $\mathscr{F}^{4}$ o has been incorporated in the notation. Similarly we write $x y \geqslant{ }_{A 0}^{*} x^{\prime} y^{\prime}$ if $x_{j} f \geqslant y_{j} g$ and $x^{\prime} f f \leqslant y_{j}^{\prime} g$, and $x_{j} f, y_{j} g, x_{j}^{\prime} f, y_{j}^{\prime} g \in \mathscr{F}_{F} A \mathrm{o}$, and, finally, $j$ is $\mathscr{F}$ Ho essential. We say that $\geqslant$ satisfies weak sign-comonotonic tradeoff consistency if there do not exist $\mathscr{F} A 0$ and $x, y, x^{\prime} y^{\prime}$ such that both $x y>{ }_{A 0}{ }^{*} x^{\prime} y^{\prime}$ and $x^{\prime} y^{\prime}{ }_{A_{0}} x y$ hold. This condition is weaker than sign-comonotonic tradeoff consistency because it only ensures tradeoff consistency within the same set $\mathscr{F}^{40}$. It implies the existence of a CPT representation with each $\mathscr{F}^{40}$, as will be demonstrated in the proof of Proposition 8.2. Actually, any condition giving a CPT representation with each set $\mathscr{F} 4 \mathrm{O}$ (i.e., an EU representation) will imply weak sign-comonotonic tradeoff consistency; hence, it could replace the latter in the proposition below. Figure 1 also shows that, for $n=2$, a CPT representation within each maximal sign-comonotonic set $\mathscr{F}^{A 0}$ does not imply CPT.

Proposition 8.2. Let $n \geq 3$, and suppose the following monotonicities are satisfied: [ $\forall i$ : $f(i) \geqslant g(i)] \Rightarrow f \geqslant g,[\forall i: f(i) \geqslant g(i), \exists i: f(i)>g(i)] \Rightarrow f>g$. Then in Statement (ii) of Theorem 6.3, not only gain-loss consistency can be dropped, but also signcomonotonic tradeoff consistency can be weakened to weak sign-comonotonic tradeoff consistency, or, alternatively, the requirement that within each sign-comonotonic set an EU representation holds.

\subsection{Stronger conditions}

The tradeoff consistency conditions can be strengthened. In CU, for revelations of * relations from (3.1) and (3.2), only the cumulative events concerning state $j$ need to be the same, not the entire rank-orderings of the states. In SDEU, for revelations of * relations from (3.1) and (3.2), only the signs of the outcomes $x, y, x^{\prime}, y^{\prime}$ need to be the same, not the entire sign-profile of prospects. In CPT the combinations of these observations hold. The following result is derived by substitution:

\section{Proposition 8.3.}

Under CU, no contradictions can arise between orderings of value differences derived from (3.1) and (3.2) whenever:

there exists a partition $\{A, B\}$ of $S \backslash\{j\}$ such that

$\left[\forall i \in A: f(i) \leqslant x, x^{\prime}, y, y^{\prime}\right]$ and $\left[\forall i \in B: f(i) \geqslant x, x^{\prime}, y, y^{\prime}\right]$,

and the same holds for $g$ instead of $f$.

Under SDEU, no contradictions can arise between orderings of value differences 
derived from (3.1) and (3.2) whenever all $x, x^{\prime}, y, y^{\prime}$ are nonnegative, or they are all nonpositive.

Under CPT, no contradictions can arise between orderings of value differences derived from (3.1) and (3.2) whenever

there exists a partition $\{A, B\}$ of $S \backslash\{\}\}$ such that

$\left[\forall i \in A: f(i) \leqslant x, x^{\prime}, y, y^{\prime}\right]$ and $\left[\forall i \in B: f(i) \geqslant x, x^{\prime}, y, y^{\prime}\right]$,

the same holds for $g$ instead of $f$, and all $x, x^{\prime}, y, y^{\prime}$

are nonnegative, or they are all nonpositive.

The tradeoff consistency condition used in Tversky and Kahneman (1992) to characterize CPT is stronger than sign-comonotonic tradeoff consistency, and weaker than the last condition above. The prospects in (3.1) and (3.2) are assumed comonotonic, but they need not be cosigned, only $x, x^{\prime}, y, y^{\prime}$ should have the same sign. Another difference is that Tversky and Kahneman impose the conditions with respect to every subpartition of the state space, whereas this paper only considers the most refined partition.

\subsection{Infinite state spaces}

Finally, we briefly discuss the extension to infinite state spaces. So we assume now that the state space $S$ is an infinite set. The outcome set $X$ is, as before, a connected topological space. Prospects are functions from $S$ to $X . S$ and $X$ can be endowed with an algebra, or a $\sigma$-algebra, and then prospects can be restricted to those that are "measurable;" this does not affect the subsequent discussion. $\mathscr{F}$ denotes the set of simple prospects, i.e., prospects taking only a finite number of outcomes. We extend the assumption of true mixedness, by assuming that there exists a simple prospect $f \in \mathscr{F}^{5}$ with both $f^{+}>\overline{0}$ and $\overline{0}>f^{-}$.

Observation 8.4. Theorem 6.3 can be modified by allowing the state space $S$ to be infinite, and then restricting the conditions and representation to the simple prospects.

Here continuity of the preference relation is imposed within each set of simple prospects, measurable with respect to a fixed finite partition of $S$. The extension to nonsimple prospects can be obtained by means of the techniques explicated in Wakker (1993b). In short, the extension to bounded prospects ${ }^{7}$ is obtained by imposing monotonicity $(f(s) \geqslant$ $g(s)$ for all $s$ then $f \geqslant g$ ), and the requirement that for each bounded prospect $f$ there exists a simple prospect $g$ such that $g^{+} \sim f^{+}, g^{-} \sim f^{-}$, and $g \sim f$. The extension to nonbounded prospects can be obtained by "truncation-continuity" conditions, but will not be discussed here any further.

A useful condition for capacities is solvability. Capacity $W$ is solvable if for all events $A$ $\subset C$ and all $W(A) \leq b \leq W(C)$ there exists an event $B$ such that $A \subset B \subset C$ and $W(B)$ 
$=b$. This condition was introduced by Gilboa (1987), who used the term convex-ranged. The preference condition to characterize solvability of $W^{+}$in the above observation is as follows. Let $\left(\alpha, A ; 0, A^{c}\right.$ ) denote the prospect that assigns outcome $\alpha$ to event $A$, and 0 to event $A^{c}$. The following condition characterizes solvability of $\mathrm{W}^{+}$, as follows from substitution:

For all events $A \subset C$, outcomes $\alpha \geqslant 0$, and prospects $f$, if $\left(\alpha, A ; 0, A^{c}\right) \leqslant f \leqslant(\alpha, C ; 0$, $\left.C^{c}\right)$, then there exists an event $B$ such that $A \subset B \subset C$, and $f \sim\left(\alpha, B ; 0, B^{c}\right)$.

Similarly, solvability of $W^{-}$is characterized by the following condition:

For all events $A \subset C$, outcomes $\alpha \leqslant 0$, and prospects $f$, if $\left(\alpha, A ; 0, A^{\mathrm{c}}\right) \geqslant f \geqslant(\alpha, C ; 0$, $\left.C^{c}\right)$, then there exists an event $B$ such that $A \subset B \subset C$, and $f \sim\left(\alpha, B ; 0, B^{c}\right)$.

\section{Characteristics of the value function}

In this section we compare the curvature of value functions, derive diminishing sensitivity and loss aversion, and characterize some parametric families of value functions. We begin by extending the classical results of Arrow-Pratt on comparability of utility functions to Cumulative Prospect Theory. It is noteworthy that these results can be obtained globally, as in Expected Utility, rather than in terms of local utility functions exploited by Machina (1982). In EU, comparisons of utility functions are interpreted in terms of risk aversion. In CPT, the comparisons of value functions are interpreted in terms of the evaluation of outcomes, not in terms of attitudes towards risk.

By means of the orderings of tradeoffs, we can compare orderings of value differences. Thus we obtain transparent characterizations of the classical properties of value functions. Furthermore, the proofs are so simple, given Lemma 6.1, that we shall not elaborate them.

For Quiggin's rank-dependent utility for decision making under risk, results concerning "risk aversion" were given by Yaari (1987), Chew, Karni, and Safra (1987), Chew (1989), Chateauneuf and Cohen (1990), and others. These papers used the condition of aversion to mean-preserving spreads, or conditions of a similar nature. At any rate, these conditions simultaneously restrict the value function and the decision weights, and do not separate between them. The latter was done in Wakker (1990b); he used, in the context of rank-dependent utility, derived tradeoffs, similar to the present paper.

We only consider the case $X=I R$ below. The results also hold for any outcome set consisting of a real interval within $I R$ that contains 0 in its interior.

Assumption 9.1. All hypotheses of Theorem 6.3 hold, and $X=I R$. The ordering of outcomes by $\geqslant$ coincides with the natural ordering $\geq$.

Obviously, the above assumption implies that the value function is strictly increasing. Now suppose there are two decision makers $\geqslant 1, \geqslant 2$ each satisfying Assumption 9.1, 
with value functions $v^{1}$ and $v^{2}$ respectively. The following results hold without additional restrictions on the gain- and loss capacities of the two decision makers. Let $>{ }_{c s}^{* 1}$ be derived from $\geqslant 1$ as above. When there is no ambiguity, we omit the subscripts $c, s$. The reversed and nonstrict relations are defined similarly; the same notations apply to $\geqslant 2$. We say that $\geqslant^{2}$ exhibits greater diminishing sensitivity than $\geqslant 1$ if there are no $\alpha \geq \beta \geq \gamma$ $\geq \delta \geq 0$ such that $\alpha \beta \leqslant{ }^{* 1} \gamma \delta$ and $\alpha \beta>{ }^{* 2} \gamma \delta$, and there are no $\alpha \leq \beta \leq \gamma \leq \delta \leq 0$ such that $\beta \alpha \leqslant{ }^{* 1} \delta \gamma$ and $\beta \alpha>>^{* 2} \delta \gamma$.

It is instructive to interpret this relation in terms of Expected Utility. The first part of the definition, pertaining to the positive domain, corresponds in Expected Utility to a greater decrease in marginal utility. The second part of the definition, pertaining to the negative domain, corresponds in Expected Utility to a greater increase in marginal utility. Recall that the basic property of the utility function in Expected Utility is concavity, or decreasing marginal utility. Consequently, utility functions are naturally compared in terms of the degree of concavity as was done by Arrow-Pratt. In contrast, the basic property of the value function in prospect theory is diminishing sensitivity giving rise to an $S$-shape function, concave for gains and convex for losses. For empirical evidence, see Tversky and Kahneman (1992). Consequently, value functions are more naturally compared in terms of the degree of diminishing sensitivity as we do in the present section.

Theorem 9.2. Under CPT, $\geqslant 2$ exhibits greater diminishing sensitivity than $\geqslant 1$ if and only if $v^{2}=\psi o v^{1}$ where $\psi$ is concave on $I R^{+}$and convex on $I R^{-}$.

As a corollary we obtain the characterization of a concave/convex value function $v$ of a single preference relation $\geqslant$ on $I R^{+}$and $I R^{-}$separately. The result follows readily from Theorem 9.2. Note that the combination of (a) and (d) below characterize an $S$-shape value function discussed above.

Corollary 9.3. Under Assumption 9.1 we have: ${ }^{8}$

(a) $\left\{v\right.$ is concave on $\left.I R^{+}\right\} \Leftrightarrow\left\{[x+\varepsilon ; y+\varepsilon]>^{*}[x ; y]\right.$ for no $\left.\varepsilon>0, x>y \geq 0\right\}$;

(b) $\left\{v\right.$ is concave on $\left.I R^{-}\right\} \Leftrightarrow\left\{[x+\varepsilon ; y+\varepsilon]>^{*}[x ; y]\right.$ for no $\left.\varepsilon>0,0 \geq x+\varepsilon>y+\varepsilon\right\}$;

(c) $\left\{v\right.$ is convex on $\left.I R^{+}\right\} \Leftrightarrow\left\{[x-\varepsilon ; y-\varepsilon]>^{*}[x ; y]\right.$ for no $\left.\varepsilon>0, x-\varepsilon>y-\varepsilon \geq 0\right\}$;

(d) $\left\{v\right.$ is convex on $\left.I R^{-}\right\} \Leftrightarrow\left\{[x-\varepsilon ; y-\varepsilon]>^{*}[x ; y]\right.$ for no $\left.\varepsilon>0,0 \geq x>y\right\}$.

Next we turn to loss aversion (see Figure 2). Loss aversion holds if the value function is steeper for losses than for gains. That is, for all $x>y \geq 0, v(x)-v(y) \leq v(-y)-v(-x)$. This property is easily characterized in EU or CU by excluding, for all $x>y \geq 0,[x ; y]$ $>^{*}[-y ;-x]$ or $[x ; y]>_{c}^{*}[-y ;-x]$ respectively. In order to characterize loss aversion in CPT, however, we must adopt a more complicated procedure because we cannot directly compare value differences of positive and negative outcomes. Suppose $x>y \geq 0$, and we find prospects $f, g$ such that $f^{+} \sim \bar{x}, g^{+} \sim \bar{y}, f^{-} \sim-\bar{x}$, and $g^{-} \sim-\bar{y}$ (see figure 2a). Then the ordering of $v(x)-v(y)$ and $v(-y)-v(-x)$ can be inferred from the preference between $f$ and $g$ :

$$
v(x)-v(y) \leq v(-y)-v(-x) \Leftrightarrow f \leqslant g .
$$




Figure 2a. The easy case where
$\mathrm{x}, \mathrm{y},-\mathrm{x},-\mathrm{y}$ can be matched by
$\mathrm{f}^{+}, \mathrm{g}^{+} \mathrm{f}^{-}$and $\mathrm{g}$ respectively.
The preference between $\mathrm{f}$ and $\mathrm{g}$
reveals the ordering of the
value difference between $\mathrm{x}, \mathrm{y}$
and between $-\mathrm{y},-\mathrm{x}$. If $\mathrm{f} \succ \mathrm{g}$ then
the former difference is larger.

Loss aversion in the small implies loss aversion in the large. Even if a difference $v(x)$ $-v(y)$ is very small, if $x, y$ are extreme and the value function is bounded, it may still be impossible to find a prospect $f$ satisfying both $f^{+} \sim \bar{x}$ and $f^{-} \sim-\bar{x}$, or a prospect $g$ satisfying both $g^{-} \sim-\bar{y}, g^{+} \sim \bar{y}$. To handle these cases, we find $\left[x_{+} ; y_{+}\right]$that matches $[x ; y]$, and $\left[y_{-} ; x_{-}\right]$that matches $[-y ;-x]$, where the new outcomes are all close to 0 (see figure 2b). Writing $\sim_{c+}^{*}$ for the intersection of $\geqslant_{c+}^{*}$ and $\leqslant_{c+}^{*}$, and $\sim_{c-}^{*}$ for the intersection of $\geqslant_{c-}^{*}$ and $\leqslant_{c-}^{*}$, and dropping subscripts, we can prove:

Proposition 9.4 (see figure 2b). Suppose Assumption 9.1 holds. Then loss aversion holds if and only if there are no $x>y \geq 0, x_{+}>y_{+} \geq 0,0 \geq y_{-}>x_{-}$, and $f$, $g$, such that:

$$
\begin{aligned}
& {[x ; y] \sim{ }^{*}\left[x_{+} ; y_{+}\right],[-y ;-x] \sim{ }^{*}\left[y_{-} ; x_{-}\right],} \\
& f^{+} \sim \bar{x}_{+}, g^{+} \sim \bar{y}_{+}, f^{-}-\bar{x}_{-}, g^{-} \sim \bar{y}_{-}, \text {and } f>g .
\end{aligned}
$$

Note that concavity of $v$ on the entire domain can be obtained by combining loss aversion with conditions (a) and (b) of Corollary 9.3.

Next we turn to the characterization of the $\log$ /power and linear/exponential families of value functions. The following results are direct corollaries of Theorems 3 and 4 in Miyamoto (1988): 
Proposition 9.5. Suppose Assumption 9.1 holds. Invariance of preference on $\mathscr{F}^{+}$with respect to multiplication of outcomes by a positive constant ${ }^{9}\left(\forall f, g \in \mathscr{F}^{+}, \sigma>0: f \geqslant\right.$ $g \Leftrightarrow \sigma f \geqslant \sigma g)$ characterizes the $\log$ /power family, i.e., either $v: x \mapsto \lambda x^{\sigma}+\tau$ for positive $\lambda \times \sigma$ and real $\tau$, or $v: x \mapsto \lambda \log (x)+\tau$ for positive $\lambda$ and real $\tau$ (the latter parametrized by $\sigma=0$ ).

Proposition 9.6. Suppose Assumption 9.1 holds. Invariance of preferences on $\mathscr{F}^{+}$with respect to addition of a positive constant ${ }^{10}$ to outcomes $\left(\forall f, g \in \mathscr{F}^{+}, \tau>0: f \geqslant g \Leftrightarrow \tau\right.$ $+f \geqslant \tau+g$ ) characterizes the linear/exponential family, i.e., either $v: x \mapsto \lambda e^{\sigma x}+\tau$ for positive $\lambda \times \sigma$ and real $\tau$, or $v: x \mapsto \lambda x+\tau$ for positive $\lambda$ and real $\tau$ (the latter parametrized by $\sigma=0$ ).

Naturally, the same results can be applied to $\mathscr{F}^{-}$to characterize the same forms of $v$ on $I R^{-}$. The parameters for $v$ on $I R^{-}$are independent of the parameters on $I R^{+}$.

\section{Appendix: proofs}

\subsection{Appendix A1. A preparation: the proportionality lemma}

The following lemma serves to derive proportionality of additive value functions:

Lemma A1 [Proportionality lemma]. Suppose $\left(V_{1}, V_{2}\right)$ are nonconstant continuous additive value functions for a binary relation $\geqslant$ ' on a connected topological product space $C \times A,\left(W_{1}, W_{2}\right)$ nonconstant continuous additive value functions for a binary relation $\geqslant "$ on a connected topological product space $C \times B$. Write $v_{1} w_{1} \geqslant{ }^{\prime *} s_{1} \mathrm{t}_{1}$ if there exist $a_{2}, b_{2} \in A$ such that $\left(v_{1}, a_{2}\right) \geqslant '\left(w_{1}, b_{2}\right),\left(s_{1}, a_{2}\right) \leqslant^{\prime}\left(t_{1}, b_{2}\right)$; if the latter preference is strict write $v_{1} w_{1}>^{\prime *} s_{1} t_{1}$. Analogously define $\geqslant * *$ and $>^{\prime *}$ on $C$ from $\geqslant "$ on $C \times B$. Suppose there do not exist $v_{1}, w_{1}, s_{1}, t_{1}$ such that $\left[v_{1} w_{1} \geqslant{ }^{*} s_{1} t_{1}\right.$ and $s_{1} t_{1}$ $\left.>^{\prime *} v_{1} w_{1}\right]$ or $\left[v_{1} w_{1}>^{\prime *} s_{1} t_{1}\right.$ and $\left.s_{1} t_{1} \geqslant " * v_{1} w_{1}\right]$. Then $V_{1}=\tau+\sigma W_{1}$ for real $\tau$ and positive $\sigma$.

Proof. Note that $\left(v_{1}, a_{2}\right) \geqslant{ }^{\prime}\left(w_{1}, a_{2}\right),\left(v_{1}, a_{2}\right) \leqslant{ }^{\prime}\left(v_{1}, a_{2}\right)$ and $\left(v_{1}, a_{2}\right) \geqslant{ }^{\prime \prime}\left(v_{1}, a_{2}\right)$ imply $\left(v_{1}, a_{2}\right) \geqslant "\left(w_{1}, a_{2}\right)$; thus $V_{1}\left(v_{1}\right) \geq V_{1}\left(w_{1}\right)$ implies $W_{1}\left(v_{1}\right) \geq W_{1}\left(w_{1}\right)$. The reversed implication holds similarly, and necessarily $V_{1}=\phi \circ W_{1}$ for a strictly increasing transformation $\phi$ that furthermore must be continuous, because it is onto from one interval to another. Now the condition in the lemma implies that $V_{1}$ differences are ordered the same way as $W_{1}$ differences whenever both the $V_{1}$ differences can be matched by $V_{2}$ differences, and the $W_{1}$ differences by $W_{2}$ differences. Next let $V_{1}\left(x_{1}\right) \in \operatorname{int}\left(V_{1}(C)\right.$ ). There exists an open interval $] V_{1}\left(v_{1}\right), V_{1}\left(y_{1}\right)$ [ around $V_{1}\left(x_{1}\right)$ so small that the difference $V_{1}\left(y_{1}\right)-V_{1}\left(v_{1}\right)$ can be matched by a $V_{2}$ difference, and also the difference $W_{1}\left(y_{1}\right)-$ $W_{1}\left(v_{1}\right)$ can be matched by a $W_{2}$ difference. Hence on this open interval around $V_{1}\left(x_{1}\right)$ 
the transformation $\phi$ preserves ordering of differences. It must be affine there. So the continuous $\phi$ has second derivative 0 at every interior point $V_{1}\left(x_{1}\right)$ of its domain, and consequently is affine.

\subsection{Appendix A2. Proof of theorem 6.3}

In this section we prove Theorem 6.3. Since necessity of the conditions is obvious, we shall only establish sufficiency, and the uniqueness results.

First we derive some elementary implications. A first implication is sign-comonotonic triple cancellation. This holds if $\left[x_{i} f \leqslant y_{i} g, x_{i}^{\prime} f \geqslant y_{i}^{\prime}{ }_{i}, x_{i} f^{\prime} \geqslant y_{i} g^{\prime} \Rightarrow x_{i}^{\prime} f^{\prime} \geqslant y_{i}^{\prime} g^{\prime}\right]$ for all $i, x$, $\ldots, g^{\prime}$ for which the four prospects are sign-comonotonic. It is the (generalized) triple cancellation condition (see, for instance, Wakker, 1989, or, for two dimensions, Fishburn, 1970, or Krantz, Luce, Suppes, and Tversky, 1971) restricted to maximal signcomonotonic subsets. Note that for a state $i$ which is inessential on the maximal signcomonotonic set containing the prospects in question, the condition trivially holds true. For other states $i$ the condition is the restriction of tradeoff consistency to the case where all orderings of tradeoffs have been derived from one same state $i$, and from one same maximal sign-comonotonic set. An implication of sign-comonotonic triple cancellation is sign-comonotonic independence. This holds if $\left[x_{i} \bar{f} \geqslant x_{i} \bar{g} \Rightarrow x_{i}^{\prime} \bar{f} \geqslant x_{i}^{\prime} \bar{g}\right]$ for all $i, x, \ldots, \bar{g}$ for which the four prospects are sign-comonotonic. It is the well-known independence condition, restricted to maximal sign-comonotonic sets. It follows from sign-comonotonic triple cancellation by substituting $f=g=\bar{f}, x=y, x^{\prime}=y^{\prime}, f^{\prime}=\bar{f}, g^{\prime}=\bar{g}$. It says that on sign-comonotonic sets, preferences are independent of a common outcome. It then follows, stronger, that:

Lemma A2.1 [Strong Sign-Comonotonic Independence]. On sign-comonotonic sets, preferences are independent of any number of common outcomes.

Proof. A typical case is illustrated in figure 3 . For the context of cumulative utility a formal argumentation has been given in Wakker (1991). The argumentation is not affected by sign-dependence.

Lemma A2.2 [Monotonicity lemma]. Weak monotonicity is satisfied, as well as strong monotonicity on each maximal sign-comonotonic set.

Proof. By Wakker (1989, Lemma VI.4.10), weak monotonicity is satisfied on $\mathscr{F}^{+}$as well as on $\mathscr{F}^{-}$. By Lemma A2.1, weak monotonicity is also satisfied within each set $\mathscr{F}^{4}$ : If $x \geqslant f(i)$, and both $x_{i} f$ and $f$ are contained in $\mathscr{F}^{A}$, then both $x$ and $f(i)$ must be nonpositive or nonnegative. Let it be the former; we then first obtain $x_{i} f^{-} \geqslant f^{-}$, and next $x_{i} f \geqslant f$ by Lemma A2.1. Finally, this implies weak monotonicity in general, because $x_{i} \geqslant f$ can be inferred from transitivity and preferences $x_{i} f \geqslant x_{i}^{1} f \geqslant \ldots \geqslant x_{i}{ }^{m} f \geqslant f$, where $x_{i} \geqslant x_{i}{ }^{1} \geqslant$ $\ldots \geqslant x_{i}^{m} \geqslant f(i)$ are chosen such that each subsequent pair is sign-comonotonic. 


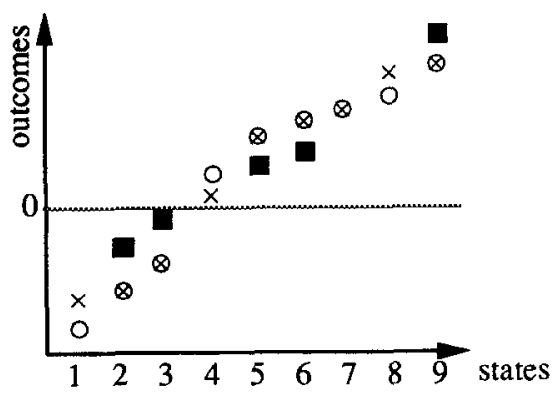

$$
\begin{aligned}
& x: \text { outcomes of prospect } f \\
& 0: \text { outcomes of prospect } g \\
& \otimes: \text { common outcomes of } \\
& \text { prospects } f \text { and } g \\
& \mathbf{~} \text { : some common outcomes } \\
& \text { are to be replaced by new } \\
& \text { ones. These new outcomes } \\
& \text { have been indicated by }
\end{aligned}
$$

Figure 3(Independence of common outcomes). $A=\{4,5, \ldots, 9\}$ and $\mathrm{o}=$ identity (outcomes "increase"). $\{2,3$, $5,6,9\}$ is the set of states at which the (common) outcomes are to be replaced. It is to be shown that this replacement does not affect preference. The common outcomes are replaced one by one, first those that are to be increased "from right to left", for, subsequently, states 9,3,2, next those that are to be decreased, "from left to right", for, subsequently, states 5, 6. These replacements do not affect the rank-ordering of the states; thus, by comonotonic independence, they indeed do not affect preference. Note that, to preserve rank-ordering, the common outcome at state 3 should be replaced prior to the common outcome at state 2 , and the common outcome at state 5 prior to that at state 6 .

Next let $x>f(i)$, where $i$ is $\mathscr{F}^{40}$ essential and both prospects are contained in $\mathscr{F}^{A 0}$. Say both $x$ and $f(i)$ are nonnegative. Then $i$ is, by comonotonic independence, also essential on $\mathscr{F}^{A} \mathrm{o}+$. Here usual "non-sign-dependent" results can be invoked, and $x_{i} f^{+}>f^{+}$ follows from Wakker (1989, Lemma VI.4.10). From strong sign-comonotonic independence (Lemma A2.1), $x_{\mathrm{i}} f>f$ follows.

Because $\geqslant$ is truly mixed we have:

There exists an event $R$ and an ordering ô such that for a prospect in $\mathscr{F} R \hat{o}$ both the positive and the negative part are not equivalent to $\overline{0}$.

Obviously, given the existence of a prospect with positive and negative part nonequivalent to $\overline{0}, R$ and $\hat{o}$ as in (A2.1) can always be found. We establish an additive representation on a two-dimensional subspace, with negative outcomes in one dimension, and positive outcomes in the other. $R^{c}$ is $\mathscr{F}^{R} \hat{o}-$ essential (compare the negative part of the above-mentioned prospect with $\overline{0}$ ), and must contain $\mathscr{F} R \hat{\alpha}-$ essential states; analogously $R$ is $\mathscr{F} R \hat{o}+$ essential, and contains $\mathscr{P} R \hat{o}+$ essential states. In the remainder of the construction of the two-dimensional representation we suppress $\mathscr{F} R \hat{o}-$ inessential states from $R^{\mathrm{c}}$, and $\mathscr{F} R \hat{o}+$ inessential states from $R$ on $\mathscr{F} R \hat{o}+$. That is, we assume that all states are essential. Suppression of inessential states affects neither preference nor the conditions that this paper imposed on preferences. We can always substitute outcomes on inessential states to stay within $\mathscr{F} R \hat{\text { ô }}$ such that all operations below are possible. Let $\hat{\mathscr{P}} \hat{\mathrm{O}(1), \hat{\mathrm{o}}(n)}$ denote the set of prospects from $\mathscr{F} R \hat{o}$ that have an outcome equivalent to 0 on coordinates $\hat{o}(2), \ldots, \hat{o}(n-1)$ (see figure 4$)$. This is isomorphic to a full twodimensional product space, of the form $X^{-} \times X^{+}$. Here sign-comonotonic triple cancellation reduces to "triple cancellation." By strong monotonicity on भुRô , both states 1 and $n$ are essential of $\mathscr{P 0}(1), \hat{o}(n)$. Hence by Wakker (1989, Theorem III.6.6(iii) and Remark A3.1) 


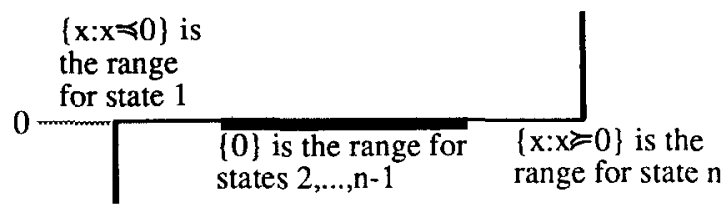

Figure 4 (The set $\left.\mathrm{F}^{\hat{o}(1), \hat{o}(n)}\right)$. Suppose $\hat{o}$ is identity. Acts from $\mathrm{F}^{\hat{o}(1), \hat{\mathrm{o}}(n)}$ "live" only on states $1, n$.

there exist continuous additive value functions, denoted by $V^{R-}$ and $V^{R+}$ in the remainder of this proof (dependency on ô is not expressed in the notation), for the preference relation on this space $\mathscr{\mathscr { H }} \hat{\mathrm{O}}(1), \hat{\mathrm{o}}(n) . V^{R-}, V^{R+}$ are "joint" (having common unit) interval scales. We may and will set $V^{R-}(0)=V^{R+}(0)=0$.

Now we establish a $\mathrm{CU}^{+}$representation on $\mathscr{F}^{+}$, and a $\mathrm{CU}^{-}$representation on $\mathscr{F}^{-}$, each up to a positive factor. First we consider $\mathscr{F}^{+}$. Because $\geqslant$is truly mixed, $\mathscr{F}^{+}$has at least one essential state, so every $\mathscr{F O}^{+}$has at least one essential state. First suppose every $\mathscr{F O}^{+}$has exactly one essential state. Now we cannot invoke Theorem 4.3 , because that required that some $\mathscr{F O}^{+}$have two essential states; neither can we invoke Theorem VI.5.1 of Wakker (1989) because that essentially needs topological separability for the present case. We shall derive the $\mathrm{CU}^{+}$representation from the function $V^{R+}$ as obtained above. Each prospect from $\mathscr{F O}+$ is equivalent to the constant prospect identical to the outcome for the essential state. By strong monotonicity the function $V^{R+}$ obtained above represents preferences over these. So then a $\mathrm{CU}^{+}$representation on $\mathscr{F}^{+}$results where on each set $\mathscr{F O}^{+}$the essential state has decision weight 1 , and the value function for gains is the continuous function $V^{R+}$.

If for some permutation o the set $\mathscr{F o ~}^{+}$has two essential states, then by Theorem $4.3 \mathrm{a}$ $\mathrm{CU}^{+}$representation on $\mathscr{F}^{+}$is obtained, which is an interval scale. We let it assign value 0 to $\overline{0}$. Subsequently we deduce from the Proportionality Lemma A1 the fact that the value function of this $\mathrm{CU}^{+}$representation is a positive multiple of $V^{R+}$, as follows. First restrict attention, for any positive nonmaximal outcomes $\mu>\nu>0$, to a twodimensional subspace of $\mathscr{F}^{+}$isomorphic to the full product space $\{\alpha \in X: 0 \leqslant \alpha \leqslant \mu\} \times$ $\{\beta \in X: \beta \geqslant \mu\}$ with both dimensions essential to get, from Lemma A1, proportionality of the value function and $V^{R+}$ separately on $\{\alpha \in X: 0 \leqslant \alpha \leqslant \mu\}$ and $\{\beta \in X: \beta \geqslant \mu\}$. Repeat this with $\nu$ instead of $\mu$. The overlap $\{\alpha \in X: \nu \leqslant \alpha \leqslant \mu\}$ shows that there indeed is one positive $\sigma$ such that the value function is $\sigma$ times $V^{R+}$.

Exactly the same procedure can be adopted to get a $\mathrm{CU}^{-}$representation on $\mathscr{F}^{-}$. The value function $v: X \rightarrow I R$ describes the value function of $\mathrm{CU}^{+}$on $X^{+}$, and of $\mathrm{CU}^{-}$on $X^{-}$. To derive continuity of the function $v$, note that it describes the value function of $\mathrm{CU}^{+}$on $X^{+}$, and of $\mathrm{CU}^{-}$on $X^{-}$. Because $v$ is continuous on $X^{+}$and on $X^{-}$, and $v(0)$ $=0$, the range of $v$ is an interval, and has no gaps; $v$ must be continuous. Below, $v$ will be rescaled for positive outcomes, i.e., there it will be multiplied by a positive constant. That will not affect its continuity.

We summarize:

$V^{R-}$ and $V^{R+}$ are positive affine transforms of $v$ on their domains; $V^{R-}, V^{R+}$, and $v$ are continuous. 
Now let $A$, o be fixed for a while. We derive an additive representation on $\mathscr{F} A$. If $A$ or $A^{c}$ is inessential on $\mathscr{F}^{40}$, then we can simply use the $\mathrm{CU}^{+}$or $\mathrm{CU}^{-}$representation. Therefore let both $A$ and $A^{\mathrm{c}}$ be essential on $\mathscr{F} A \mathrm{o}$. Then on $\mathscr{F}^{A 0}$ there are at least two essential states. The condition of sign-comonotonic generalized triple cancellation, as implied by sign-comonotonic tradeoff consistency, simply reduces to generalized triple cancellation on $\mathscr{F A o}$. Generalized triple cancellation was used in Wakker (1989, Theorem III.6.6(iii) and Remark A3.1) to characterize additive representability on full product sets. The set $\mathscr{F}^{40}$, however, is not a full product set. It is a product of two (rank-ordered sets =) maximal comonotonic subsets of a product set, one isomorphic to $\mathscr{F A o}$, the other to $\mathscr{F}^{A 0+}$; we shall use the notations $\mathscr{F}_{40-}, \mathscr{F}^{40+}$ also for these subsets. We suppress for the time being the states inessential on $\mathscr{F}^{A}$. Then, by Theorem C.6 and Remark C.7 of Chateauneuf and Wakker (1991), an additive representation is obtained on $\mathscr{F} 4$; we may let the additive value functions assign 0 to 0 . On $\mathscr{F A O}+$ the additive representation is proportional to $\mathrm{CU}^{+}$. This follows from the uniqueness result of Theorem 4.3 if $\mathscr{F}^{40+}$ has two or more essential states. If $\mathscr{F}^{40+}$ has only one essential state the argument is similar to the derivation of (A2.2), as follows. We again suppress the $\mathscr{F} A \mathrm{o}+$ inessential states in $A$, and restrict attention to prospects from $\mathscr{F} A \mathrm{Ao}$ that are constant (nonpositive) on $A^{c}$. This yields a two-dimensional space, and by the Proportionality Lemma A1 the additive value function on the two-dimensional space for gains is proportional to $V^{R+}$, and therefore to the value function $v$, and so to $\mathrm{CU}^{+}$. Similarly, on $\mathscr{F F}^{4} \mathrm{o}-$ the additive representation is proportional to $\mathrm{CU}^{-}$. We conclude:

There exists $\lambda_{A \mathrm{o}}^{+}>0$ s.t. $f \mapsto \lambda_{A \mathrm{o}}^{+} \mathrm{CU}^{+}\left(f^{+}\right)+\mathrm{CU}^{-}\left(f^{-}\right)$represents $\geqslant$ on $\mathscr{F F}^{40}$.

Thus we have obtained a CPT representation on each $\mathscr{F}^{A 0}$. Note that $\lambda_{A \mathrm{o}}^{+}>0$ can be chosen freely if $\mathscr{F F}^{A 0}+$ or $\mathscr{F}^{40-}$ is inessential. Below we set then $\lambda_{A \mathrm{o}}^{+}=1$. The next step is to fit all these representations together into one global CPT functional. Only here gainloss consistency is used.

Lemma A2.3. There exists a CPT functional on the entire $\mathscr{F}$ that is representing within each $\mathscr{F}^{A 0}$, with the value function $v$ continuous.

Proof. For the time being we only require the functions discussed below to be representing within each $\mathscr{F}^{4 \mathrm{Ao}}$. We rescale the $\mathrm{CU}^{+}$representation such that $\lambda_{R \hat{\mathrm{o}}}^{+}=1$ (for $R$ see (A2.2); dependence on ô is not expressed in notation there). This can be obtained by multiplying the "old" $\mathrm{CU}^{+}$representation (so $v$ on $X^{+}$) by $\lambda_{R \hat{o}}^{+}$and by dividing each "old" $\lambda_{A \mathrm{o}}^{+}$by $\lambda_{R \hat{o}}^{+}$and henceforth calling the latter quotient $\lambda_{A \mathrm{o}}^{+}$. If $A$ or $A^{c}$ is inessential then we set $\lambda_{A \mathrm{o}}^{+}=1$. We prove now that all $\lambda_{A \mathrm{o}}^{+}$are 1 . Suppose that both $A$ and $A^{c}$ are $\mathscr{F}^{A o}$ essential. By continuity we can straightforwardly construct $f \in \mathscr{F}^{A o}, f^{r} \in \mathscr{F}^{R o}$, such that:

$$
\overline{0}<f^{+} \sim f^{r+}, f^{-} \sim f^{r^{-}}<\overline{0} \text {, and } f^{r} \sim \overline{0} .
$$


By gain-loss consistency, also $f \sim \overline{0}$. Substituting the representations within $\mathscr{F} R \hat{0}$ and $\mathscr{F} A \mathrm{o}$, we get $\mathrm{CU}^{+}\left(f^{+}\right)+\mathrm{CU}^{-}\left(f^{-}\right)=0$, and $\lambda_{A 0}^{+} \mathrm{CU}^{+}\left(f^{+}\right)+\mathrm{CU}^{-}\left(f^{-}\right)=0$. Further $\mathrm{CU}^{+}$ $\left(f^{+}\right)=\mathrm{CU}^{+}\left(f^{+}\right)>0$ and $\mathrm{CU}^{-}\left(f^{-}\right)=\mathrm{CU}^{-}\left(f^{-}\right)<0$. These equalities together imply $\lambda_{A \mathrm{o}}^{+}=1$. Continuity of $v$ was established in (A2.2); as pointed out above (A2.2), the rescaling of $v$ on $X^{+}$does not affect continuity.

\section{Lemma A2.4. CPT holds.}

Proof. It remains to be shown that the CPT functional, as constructed above, is representing. Let $f \in \mathscr{F} A \circ$. Say $f \geqslant \overline{0}$. We can, by continuity, find $g^{+} \in \mathscr{F} A 0$ and $x \in X$, such that $f \sim g^{+} \sim \bar{x}$. Then, because the first pair is contained in $\mathscr{F}^{40}$, the second in $\mathscr{F}^{+}$, and CPT is representing within $\mathscr{F}^{A \mathrm{o}}$ and $\mathscr{F}^{+}, \mathrm{CPT}(f)=\mathrm{CPT}\left(g^{+}\right)=\mathrm{CPT}(\vec{x})$ follows. Thus any prospect has a CPT value identical to the "certainty-equivalent," i.e., the outcome equivalent to it. Because CPT orders certain outcomes according to preference, it is representing on the entire $\mathscr{F}$.

Thus the proof of the implication (ii) $\Rightarrow$ (i) in Theorem 6.3 has been completed. Next we turn to the uniqueness result. If each $\mathscr{F}^{+}$has exactly one essential state, then $W^{+}$ must be such that for each $\mathscr{F O}^{+}$that single essential state has decision weight one. This uniquely determines $W^{+}$. If $\mathscr{F}^{+}$has two or more essential states, then uniqueness of $W^{+}$ follows from Theorem 4.3. Analogously uniqueness of $W^{-}$follows. Given this uniqueness of $W^{+}$and $W^{-}$, the uniqueness of $V^{R-}$ and $V^{R+}$ in (A2.2) up to common scale and location together with $v(0)=0$ determine $v$ on $I R^{+}$and $v$ on $I R^{-}$up to a common scale. Because $v$ can be multiplied by any positive constant, it indeed is a ratio scale.

\subsection{Appendix A3. Proofs of results of section 8}

As a preparation for the proof of Observation 8.1, we list below the conditions of Theorem 6.3 except gain-loss consistency:

Assumption A3.1. The Structural Assumption 2.1 holds, and the truly-mixed case holds. Moreover, weak ordering, continuity, and sign-comonotonic tradeoff consistency hold.

In the proof of Theorem 6.3 in Appendix A2, gain-loss consistency is only used in the proof of Lemma A2.3, so the results obtained up to that point hold under Assumption A3.1. They include:

Lemma A3.2. under Assumption A3.1 a CU ${ }^{+}$representation exists on $\mathscr{F}^{+}$, and a $\mathrm{CU}^{-}$representation exists on $\mathscr{F}^{-}$. Within each set $\mathscr{F}^{4 \mathrm{o}}$ there exists a representation of the form $f \mapsto \lambda_{A 0}^{+} \mathrm{CU}^{+}\left(f^{+}\right)+\mathrm{CU}^{-}\left(f^{-}\right)$.

Note that $\lambda_{A \mathrm{o}}^{+}$is uniquely determined except in trivial cases of inessentiality of $A$ or $A^{c}$. In the latter cases we set $\lambda_{A \mathrm{o}}^{+}=1 . \mathscr{F}^{A \mathrm{o}}$ and $\mathscr{F B O}^{\prime}$ are directly gain-loss linked if there exists 
an $f \in \mathscr{F} A \mathrm{o} \cap \mathscr{F B O}^{\prime}$ such that $f^{+}>\overline{0}, \overline{0}>f^{-}, f \sim 0$. The transitive closure of the directly gain-loss linked relation is called the (indirectly) gain-loss linked relation. The following lemma follows from substitution. The subsequent lemma is proven as Theorem 6.3 , with Lemma A2.3 derived from Lemma A3.3.

Lemma A3.3. Suppose $\mathscr{F} A 0$ and $\mathscr{F H}^{\circ 0^{\prime}}$ are gain-loss linked. Then $\lambda_{A \mathrm{o}}^{+}=\lambda_{B \mathrm{o}^{\prime}}^{+}$can be chosen in Lemma A3.2.

Lemma A3.4. Suppose $\mathscr{F} A 0$ and $\mathscr{F} B 0^{\prime}$ are gain-loss linked whenever both $A$ and $A^{c}$ are essential on $\mathscr{F} A \mathrm{o}$, and $B$ and $B^{c}$ on $\mathscr{F B O}^{\prime}$. Then in Theorem 6.3 the condition of gainloss consistency can be dropped.

Now we are ready to prove Observation 8.1.

Proof of Observation 8.1. Denote by $[\mathrm{o}(1), \ldots, \mathrm{o}(k), \mid, \mathrm{o}(k+1), \ldots, \mathrm{o}(n)]$ the set $\mathscr{F} A \mathrm{o}$ with $A=\{\mathrm{o}(k+1), \ldots, \mathrm{o}(\mathrm{n})\}$; here $1 \leq k \leq n-1$ is implicit. This set is directly gain-loss linked to every set obtained by moving $\mid$ to the left or right, as long as $\mid$ is between $\mathrm{o}(1)$ and $\mathrm{o}(n)$ : Take, by monotonicity and continuity, a prospect $f \sim \overline{0}$ with $f(\mathrm{o}(1))<0 \sim f(\mathrm{o}(2)) \sim \ldots \sim f(\mathrm{o}(n-1)) \sim 0<f(\mathrm{o}(n))$. A directly gain-loss linked set also results from interchanging states that are to the left of the $\mid$ symbol, or to the right of the symbol: one takes a prospect $f \sim \overline{0}$ that is constant on $\{\mathrm{o}(1), \ldots, \mathrm{o}(k)\}$ and on $\{\mathrm{o}(k+1), \ldots, \mathrm{o}(n)\}$. Thus moving the symbol | leads to new gain-loss linked sets, as well as interchanging states at one side of $\mid$. By these operations one sees that all sets $\mathscr{F}^{A}$ for $\varnothing \neq A \neq S$ are mutually gain-loss linked. Lemma A3.4 now gives the desired result.

Proof of Proposition 8.2. Strong sign-comonotonic independence and monotonicity are derived as in Lemmas A2.1 and A2.2. We begin by deriving a CPT representation within each maximal sign-comonotonic subset $\mathscr{F} A$ o. First consider the case where $A=S$; i.e., consider the set $\mathscr{F O}^{+}$. On this set continuity, weak ordering, monotonicity, and generalized triple cancellation (see beginning of Appendix A2) are satisfied. Hence by Wakker (1991a, Theorem 3.2) there exists a continuous additive representation for $\geqslant$ on $\mathscr{F O}^{+}$that is possibly extended real-valued: it might assign value infinite to a maximal prospect, and value minus infinite to a prospect (e.g., $\overline{0})$ that is minimal on the domain under consideration. Consider outcomes $0<v<\mu<\sigma$, and the two-dimensional substructures $\{x \in X: \nu \leqslant x \leqslant \mu\} \times\{x \in X: \mu \leqslant x \leqslant \sigma\} \times\{\sigma\} \times \ldots \times\{\sigma\}$ and $\{x \in X$ : $0<x \leqslant \nu\} \times\{x \in X: \mathrm{v} \leqslant x \leqslant \mu\} \times\{\sigma\} \times \ldots \times\{\sigma\}$ of $\mathscr{F o}+$. By the proportionality lemma $\mathrm{A} 1$, and sign-comonotonic tradeoff consistency, the first-state additive value function and the second-state additive value function are linear transforms of each other on $\{x \in X: \nu \leqslant x \leqslant \mu\}$. This holds for all $0<v \leqslant \mu$ with $\mu$ nonmaximal, and also for all pairs of additive value functions. By Wakker (1993a, Proposition 3.5) the additive representation is real-valued after all. By continuity, the additive value functions must also be related by linear transformations at the outcome 0 as well as at maximal outcomes. We can let the additive value functions assign value 0 to outcome 0 . So a CPT representation 
with a continuous value function has been obtained on $\mathscr{F O}^{+}$. Similarly a CPT representation with a continuous value function is obtained on $\mathscr{g}^{-}$.

Next consider a set $\mathscr{F} A \mathrm{o}$ with $\emptyset \neq A \neq S$. This set is a product of two rank-ordered sets. By monotonicity, all states are essential on this set. Weak ordering, continuity, monotonicity, and generalized triple cancellation are satisfied. Hence by Chateauneuf and Wakker (1991, Theorem C.6 and Remark C.7) there exists a continuous additive representation for $\geqslant$ on $\mathscr{F}^{A}$. If $A$ contains two or more states, then the ("gains") additive value functions related to the states of $A$ can be taken proportional by a reasoning as in the discussion of $\mathscr{F O}^{+}$above. Similarly, if $A^{c}$ contains two or more states, then the ("losses") additive value functions related to the states of $A^{c}$ can be taken proportional. Again a CPT representation has resulted on $\mathscr{F}^{A 0}$.

So on each set $\mathscr{F}^{A \mathrm{o}}$ a CPT $(=\mathrm{EU})$ representation exists, with a value function $v^{A \mathrm{o}}$. By the analogue of Lemma $6.1,>_{A 0}^{*}$ and $\geqslant *{ }_{A 0}^{*}$ still reveal orderings of $v^{A 0}$ differences. It suffices to show that for any two different $\mathscr{F}^{A 0}$ and $\mathscr{F}^{B} 0^{\prime}, v^{A 0}$ on $X^{+}$is a positive multiple of $v^{B 0^{\prime}}$, also $v^{A 0}$ on $X^{-}$is a positive multiple of $v^{B 0^{\prime}}$. Then $x y>{ }_{A o}^{*}$ $x^{\prime} y^{\prime}$ and $x^{\prime} y^{\prime} \geqslant_{B 0^{\prime}}^{*} x y$ cannot both occur, also if $A \neq B$, and/or $o \neq 0^{\prime}$. In other words, sign-comonotonic tradeoff-consistency is then satisfied, and we can invoke Observation 8.1.

Suppose that $\mathscr{F} A \mathrm{o}$ and $\mathscr{F} B \mathrm{o}^{\prime}$ are directly gain-loss linked. Let $f$ be a prospect in their intersection with $f \sim \overline{0}, f^{+}>\overline{0}, f^{-}<\overline{0}$. Consider the two-dimensional subspace of prospects that are 0 where $f$ is 0 , that are constant positive-or-zero where $f$ is positive, and constant negative-or-zero where $f$ is negative. This two-dimensional subspace is a full product set; it is contained in both $\mathscr{F}^{A \mathrm{o}}$ and $\mathscr{F}^{\circ \mathrm{O}^{\prime}}$; and both dimensions are essential. Both the representation of $\mathscr{F} A \mathrm{o}$ and that of $\mathscr{F}^{B \mathrm{~B}^{\prime}}$ are additive representations on the subspace. By standard uniqueness results this implies that the value functions of $\mathscr{F A o}$ and $\mathscr{F} B \mathrm{o}^{\prime}$ are proportional on $X^{+}$, as well as on $X^{-}$. We saw already in the proof of Observation 8.1 that all nondegenerate sets $\mathscr{F}^{A} \mathrm{o}$ and $\mathscr{F}^{\mathrm{B} \mathrm{Bo}^{\prime}}$ are indirectly gain-loss linked. This completes the proof.

Proof of Observation 8.4. Suppose the preference conditions in Statement (ii) of Theorem 6.3 hold on $\mathscr{F}$. For every finite partition of $S$, fine enough to satisfy true-mixedness, Theorem 6.3 can be applied to the prospects measurable with respect to that partition (continuity of the preference relation is imposed within each such set of prospects). Next, the CPT representations can be fit together for different partitions, by considering common refinements of these partitions. This gives one overall CPT representation on $\mathscr{F}$. Also the uniqueness results of Theorem 6.3 are the same here. Necessity of the preference conditions in Theorem 6.3 is straightforward.

\subsection{Appendix A4. Proof of proposition 9.4}

Proof of Proposition 9.4. First suppose loss aversion holds, and suppose $x, \ldots, g$ are as described in the proposition. The preference $f>g$ implies CPT $\left(f^{+}\right)-\operatorname{CPT}\left(g^{+}\right)>$ $\operatorname{CPT}\left(g^{-}\right)-\operatorname{CPT}\left(f^{-}\right)$. Hence CPT $\left(\bar{x}_{+}\right)-\operatorname{CPT}\left(\bar{y}_{+}\right)>\operatorname{CPT}\left(\bar{y}_{-}\right)-\operatorname{CPT}\left(\bar{x}_{-}\right)$, i.e., $v\left(x_{+}\right)-v\left(y_{+}\right)>v\left(y_{-}\right)-v\left(x_{-}\right)$. By Lemma 6.1 this implies $v(x)-v(y)>v(-y)-$ $v(-x)$, contradicting loss aversion. 
Next suppose the condition of the lemma holds. To derive is loss aversion. Because of true-mixedness there is an $\tilde{f} \in F^{R}$ such that $\tilde{f}^{+}>\overline{0}$ and $\overline{0}>\tilde{f}^{-}$. By continuity there exists $\mu>0$ such that for each $0 \leq x \leq \mu$ and $-\mu \leq-y \leq 0$ there is an $f \in F^{R}$ with $f^{+}$ $\sim \bar{x}$ and $f^{-} \sim-\bar{y}$. Also $\mu$ can be taken so small that for all $\operatorname{such} x, y$ we have $[x ; y] \sim{ }_{c+}^{*}$ $[x ; y],[-y ;-x] \sim{ }_{c}^{*}-[-y ;-x]$. For the latter, seemingly trivial, relations it must be possible to find proper sign-comonotonic prospects as in (3.1) and (3.2). By continuity, for $\mu$ sufficiently small, this is possible. Thus for each $\mu \geq x>y \geq 0$ there are prospects $f, g$ such that $f^{+} \sim \bar{x}, g^{+} \sim \bar{y}, f^{-} \sim-\bar{x}, g^{-} \sim-\bar{y}$. The condition in the proposition now implies $f \leqslant g$. By substitution, $v(x)-v(y) \leq v(-y)-v(-x)$. On the interval $[-\mu, \mu]$ loss aversion holds.

Next consider a general outcome $z>0$. By continuity we can take an open interval $I$ around $z$ within $I R^{+}$so small that for all $x, y$ from $I$ there exist $x_{+}, y_{+}, y_{-}, x$ - from the interval $[-\mu, \mu]$ with $[x ; y] \sim{ }_{c+}^{*}\left[x_{+} ; y_{+}\right],[-y ;-x] \sim{ }_{c-}^{*}\left[y_{-} ; x_{-}\right]$. As in the beginning of this proof, $v\left(x_{+}\right)-v\left(y_{+}\right) \leq v\left(y_{-}\right)-v\left(x_{-}\right)$follows; by repeated application of (6.7), $v(x)-v(y) \leq v(-y)-v(-x)$ follows. So for each outcome $z>0$ there exists an open neighborhood $I$ such that for prospects with outcomes in this neighborhood loss aversion holds. This implies loss aversion "globally."

\section{Notes}

1. It has also been called the rank-dependent model. We use the adjective cumulative to refer to the model, and we use rank-dependence and sign-dependence to refer to specific patterns of choices (see Examples 1.1 and 1.2).

2. Properties of the decision weights will be characterized in a future paper.

3. Because the standard interpretation of expected utility refers to acts rather than to prospects, the versions of expected utility and cumulative utility investigated in this paper should perhaps be called incremental expected utility and incremental cumulative utility. For brevity we omit the adjective incremental and hope that this will cause no confusion. Also we write expected utility rather than subjective expected utility.

4. Given continuity and weak ordering; see Theorem 3.3

5. $A^{c}$ denotes the complement of $A$.

6. Note that comonotonic prospects are cosigned if there exists a state $i$ with $f(i)=g(i)=0$.

7. A prospect is bounded if there is a lower bound outcome, dispreferred to all outcomes of the prospect, and, similarly, an upper bound outcome.

8. Note that subscripts $c, s$ have been dropped from the $>$ * notations.

9. Known in EU as constant relative risk aversion. In the present context the term preference homogeneity seems more appropriate.

10. Known in EU as "constant (absolute) risk aversion." In the present context the term translation independence may be used.

\section{References}

Chateauneuf, A. (1990). "On the Use of Comonotonicity in the Axiomatization of EURDP Theory for Arbitrary Consequences." Extended abstract presented at Fifth International Conference on the Foundations and Applications of Utility, Risk and Decision Theory (FUR-90), CERMSEM, University of Paris I.

Chateauneuf, A. and M. Cohen. (1990). "Risk Seeking with Diminishing Marginal Utility in a Non-Expected Utility Model," CERMSEM, University of Paris I.

Chateauneuf, A. and P.P. Wakker. (1991). "From Local to Global Additive Representation," Journal of Mathematical Economics, forthcoming. 
Chew, S.H. (1989). "The Rank-Dependent Quasilinear Mean." Unpublished manuscript, Department of Economics, University of California, Irvine.

Chew, S.H., and L.G. Epstein. (1989). "Axiomatic Rank-Dependent Means," Annals of Operations Research 19, 299-309.

Chew, S.H., E. Karni, and Z. Safra. (1987). "Risk Aversion in the Theory of Expected Utility with Rank Dependent Probabilities," Joumal of Economic Theory 42, 370-381.

Chew, S.H. and P.P. Wakker. (1991). "Generalizing Choquet Expected Utility by Weakening Savage's SureThing Principle," Irvine Research Unit in Mathematical Behavioral Sciences, MBS 91-16, University of California, Irvine.

Choquet, G. (1953-4). "Theory of Capacities," Annales de l'Institut Fourier (Grenoble), 5, 131-295.

Fishburn, P.C. (1988). Nonlinear Preference and Utility Theory. Baltimore: Johns Hopkins University Press.

Gilboa, I. (1987). "Expected Utility with Purely Subjective Non-Additive Probabilities," Journal of Mathematical Economics 16, 65-88.

Green, J. and B. Jullien. (1988). "Ordinal Independence in Non-Linear Utility Theory," Journal of Risk and Uncertainty 1, 355-387. (Erratum 2, 1989, 119.)

Kahneman, D. and A. Tversky. (1979). "Prospect Theory: An Analysis of Decision under Risk," Econometrica 47, 263-291.

Krantz, D.H., R.D. Luce, P. Suppes, and A. Tversky, (1971). Foundations of Measurement, vol. 1. (Additive and Polynomial Representations). New York: Academic Press.

Kelley, J.L. (1955). General Topology. London: Van Nostrand.

Luce, R.D., and P.C. Fishburn. (1991). "Rank- and- Sign Dependent Linear Utility Models for Finite FirstOrder Gambles," Journal of Risk and Undertainty 4, 29-59.

Machina, M.J. (1982). "Expected Utility' Analysis without the Independence Axiom," Econometrica 50, 277-323.

Markowitz, H. (1952). "The Utility of Wealth," Joumal of Political Economy, 60, 151-158.

Miyamoto, J.M. (1988). "Generic Utility Theory: Measurement Foundations and Applications in Multiattribute Utility Theory," Joumal of Mathematical Psychology 32, 357-404.

Quiggin, J. (1982). "A Theory of Anticipated Utility," Joumal of Economic Behavior and Organization 3, 323-343.

Schmeidler, D. (1989). "Subjective Probability and Expected Utility without Additivity," Econometrica 57, 571-587.

Segal, U. (1993). "The Measure Representation: A Correction," Joumal of Risk and Uncertainty 6, 99-107.

Starmer, C., and R. Sugden. (1989). "Violations of the Independence Axiom in Common Ratio Problems: An Experimental Test of Some Competing Hypotheses," Annals of Operations Research 19, 79-101.

Tversky, A., and D. Kahneman. (1992). "Advances in Prospect Theory: Cumulative Representation of Uncertainty," Joumal of Risk and Uncertainty 5, 297-323.

Tversky, A., S. Sattath, and P. Slovic. (1988). "Contingent Weighting in Judgment and Choice," Psychological Review 95, 371-384.

Wakker, P.P. (1989). Additive Representations of Preferences: A New Foundation of Decision Analysis. Dordrecht: Kluwer Academic Publishers.

Wakker, P.P. (1990a). "Under Stochastic Dominance Choquet-Expected Utility and Anticipated Utility are Identical," Theory and Decision 29, 119-132.

Wakker, P.P. (1990b). "Separating Marginal Utility and Probabilistic Risk Aversion." Theory and Decision, forthcoming.

Wakker, P.P. (1991). “Additive Representations on Rank-Ordered Sets. I. The Algebraic Approach,” Joumal of Mathematical Psychology 35, 501-531.

Wakker, P.P. (1993a). "Additive Representations on Rank-Ordered Sets. II. The Topological Approach," Journal of Mathematical Economics, 22,1-26.

Wakker, P.P. (1993b). "Unbounded Utility for Savage's 'Foundations of Statistics', and other Models," Mathematics of Operations Research, 18, 446-4855.

Wakker, P.P. (1993c). "Counterexamples to Segal's Measure Representation Theorem," Joumal of Risk and Uncertainty 6, 91-98.

Weymark, J.A. (1981). "Generalized Gini Inequality Indices," Mathematical Social Sciences 1, 409-430.

Yaari, M.E. (1987). "The Dual Theory of Choice under Risk," Econometrica 55, 95-115. 